\title{
How to Improve Quality Assurance in Fluorometry: Fluorescence-Inherent Sources of Error and Suited Fluorescence Standards
}

\author{
U. Resch-Genger, ${ }^{1,6}$ K. Hoffmann, ${ }^{1}$ W. Nietfeld, ${ }^{2}$ A. Engel, ${ }^{3}$ J. Neukammer, ${ }^{4}$ \\ R. Nitschke, ${ }^{5}$ B. Ebert, ${ }^{4}$ and R. Macdonald ${ }^{4}$
}

Received October 15, 2004; accepted January 28, 2005

\begin{abstract}
The scope of this paper is to illustrate the need for an improved quality assurance in fluorometry. For this purpose, instrumental sources of error and their influences on the reliability and comparability of fluorescence data are highlighted for frequently used photoluminescence techniques ranging from conventional macro- and microfluorometry over fluorescence microscopy and flow cytometry to microarray technology as well as in vivo fluorescence imaging. Particularly, the need for and requirements on fluorescence standards for the characterization and performance validation of fluorescence instruments, to enhance the comparability of fluorescence data, and to enable quantitative fluorescence analysis are discussed. Special emphasis is dedicated to spectral fluorescence standards and fluorescence intensity standards.
\end{abstract}

KEY WORDS: Fluorescence; standard; calibration; microarray; in vivo imaging; flow cytometry.

\section{INTRODUCTION}

Fluorescence techniques are widely used as analytical tools and detection methods in different areas such as material sciences, environmental analysis, bioanalysis, molecular genetics, cell biology, medical diagnostics, and drug screening [1-5]. Commonly recognized advantages of fluorescence are its high sensitivity that allows even the detection of single molecules, its intrinsic selectivity concerning experimental parameters such as excitation and emission wavelength as well as fluorescence lifetime and (de)polarization [6], its ease of use,

\footnotetext{
${ }^{1}$ Federal Institute for Materials Research and Testing, Working Group Optical Spectroscopy, Division I.3, BAM, Berlin, Germany.

${ }^{2}$ Max Planck Institute for Molecular Genetics, Berlin, Germany.

${ }^{3}$ Schott AG, Mainz, Germany.

${ }^{4}$ Physikalisch-Technische Bundesanstalt, Dept. 8.3, PTB, Berlin, Germany.

${ }^{5}$ Life Imaging Center, Institute of Biology I, Developmental Biology, Freiburg, Germany.

${ }^{6}$ To whom correspondence should be addressed. E-mail: ute.resch@ bam.de
}

non- or minimally invasive character, and the remote accessibility of signals employing conventional optics and fiber optics. Depending on the specific application, fluorescence measuring systems strongly differ in instrumental design, i.e. use of optical components and detection systems as well as in measurement geometries including sample containers/formats for the investigation of fluorescent samples. Typical instruments range from conventional monochromator- or filter-type fluorometers and microplate readers over fluorescence microscopes, flow cytometers, and systems for (real time) polymerase chain reaction (rt-PCR) as well as devices for molecular imaging to microarray scanners for DNA or protein chips and high throughput screening systems [5,7-10]. Independent of the technique and particular instrumentation applied, a major drawback of fluorescence measurements is the demand to remove instrument-specific effects from the measured raw data that otherwise limit the comparability of fluorescence data across instruments, laboratories, and over time [1-4,11,12]. Further fluorescenceinherent problems are related to difficulties to accurately measure absolute fluorescence intensities and hence to 
realize true quantitative measurements [13]. In addition, quantitative fluorometry based on measurements of relative fluorescence intensities is hampered by the sensitivity of the absorption and fluorescence properties of most chromophores to their microenvironment that results in environment-specific absorption and emission spectra as well as molar absorption coefficients, fluorescence quantum yields, and fluorescence lifetimes, respectively. These problems and disadvantages render quality control difficult and make standardization even more important.

The improvement in quality assurance for fluorescence techniques critically relies on the development of internationally accepted and purpose-fit guidelines for instrument characterization and performance of fluorescence measurements which are barely available at present. The former, however, depends on the availability of commercial, easy-to-operate, and reliable fluorescence standards, that take special requirements of each particular fluorescence technique into account, and that are preferably certified in view of traceability and accreditation needs. Within the context of this paper, fluorescence standards refer to chromophore-based reference materials for fluorometric quantities, which are designed for specific fluorescence applications. This includes both instrument-type standards for the calibration and performance validation of fluorescence instruments as well as more applicationspecific standards for instance to relate chemical concentration to instrument response, i.e. typically to fluorescence intensity. Furthermore, for certain fluorescence techniques, the evaluation of commonly used software routines like e.g. fitting routines for the analysis of timeresolved fluorescence data as well as software for the statistical analysis of imaging data and the analysis of microarray experiments is gaining importance and will eventually be mandatory.

At present, these needs are poorly met for fluorescence techniques, despite of their widespread use and the extensive literature dedicated to the characterization of fluorescence measuring equipment and potential fluorescence standards [1-4,14-18], as well as the globalizationinduced anchor role of traceability and accreditation [19]. The latter initiated for instance standardization in closely related fields like UV/Vis/IR spectrophotometry [20-23] and colorimetry [24-27]. Up to now, there exist only a very limited number of recommendations on the characterization of fluorescence instrumentation and performance of fluorescence measurements like the determination of the linear range of detection systems and the limit of detection [28-30]. Furthermore, with the exception of flow cytometry [31-34], surveys on instrument calibration and comparability of fluorescence data have been barely performed $[35,36]$. In addition, available or recommended fluorescence standards are often of insufficient quality with respect to their analytical and spectroscopic characterization and stability as well as reproducibility or are even not purpose-fit. For a long time, only a single certified reference material [37], the traceable emission standard quinine sulfate dihydrate (SRM 936a), developed by the National Institute of Standards and Technology (NIST) in the late 1970s, was commercially available [38]. This reference material enables the determination of the spectral responsivity of fluorescence instruments in the spectral range from about 395 to $565 \mathrm{~nm}$. Only recently, a new though not certified reference material, RM8640, i.e. microspheres with varying amounts of immobilized fluorescein molecules was released by NIST for flow cytometry thereby aiming at the establishment of a reference scale for fluorescence intensity [39].

To underline the importance of an improved quality control for the majority of fluorescence techniques, in this review, problems and fluorescence-inherent sources of error are revealed exemplary for macro- and microfluorometry, fluorescence microscopy, flow cytometry, fluorescence-based microarray technology, and in vivo fluorescence spectroscopy/imaging. The aim is to highlight the need for internationally accepted guidelines for instrument characterization and performance of typical fluorescence measurements in combination with the need for better-suited and certified fluorescence standards thereby improving the reliability of fluorescence data. This could eventually broaden the application of fluorescence methods, especially in areas like medical diagnostics that require instrument performance validation (IPV), standardization of measurements, and comparability of data for the acceptance of analytical methods. Accordingly, the scope of this article is to discuss purpose-fit solutions to these problems with special emphasis dedicated to specific existing fluorescence standards and the need for and requirements on improved systems rather then to give a comprehensive overview of the literature on the characterization of fluorescence instruments and potential reference materials.

\section{FLUORESCENCE-INHERENT SOURCES OF SYSTEMATIC ERRORS}

The majority of fluorescence-based measuring or detection methods relies on the assumption that the recorded fluorescence intensity is proportional to the concentration of the fluorophore(s) in the sample. However, independent of fluorescence technique and type of measurement, i.e. spectrally resolved or integrated over a small wavelength interval, the generated fluorescence signal is 
affected by both the fluorescent analyte(s) and the instrument. Properties of the sample that influence the magnitude of the resulting fluorescence signal are the fluorophore's absorptance at the excitation wavelength (interval) and the fluorescence quantum yield $[40,41]$. The former is nonlinearly linked to absorbance and thus molar absorption coefficient and concentration by the BeerLambert law. Absorptance and quantum yield as well as the spectral shape and position of a chromophore's absorption, excitation and emission spectrum can strongly depend on the dye's microenvironment, e.g. the polarity of the surrounding solvent/matrix molecules, $\mathrm{pH}$ as well as the concentration of certain ions in the sample $[1-5,13]$. Furthermore, specific solvent-solute or matrix-solute interactions can play a role. From the instrument side, the time-, wavelength-, and polarization-dependent spectral irradiance of the excitation channel reaching the sample and the spectral responsivity of the emission channel influence the magnitude of the recorded fluorescence intensity and the shape of the measured fluorescence spectra, respectively [40,42]. To illustrate the distorting effects of the spectral characteristics of fluorescence measuring systems, the normalized spectral responsivities of common types of fluorescence instruments are exemplary shown in Fig. 1. These curves have been determined with a spectral radiance transfer standard [40]. Accordingly, aside from spectral irradiance-related effects, measured emission intensities or spectra represent truly sample-related data that are superimposed by the spectral responsivity of

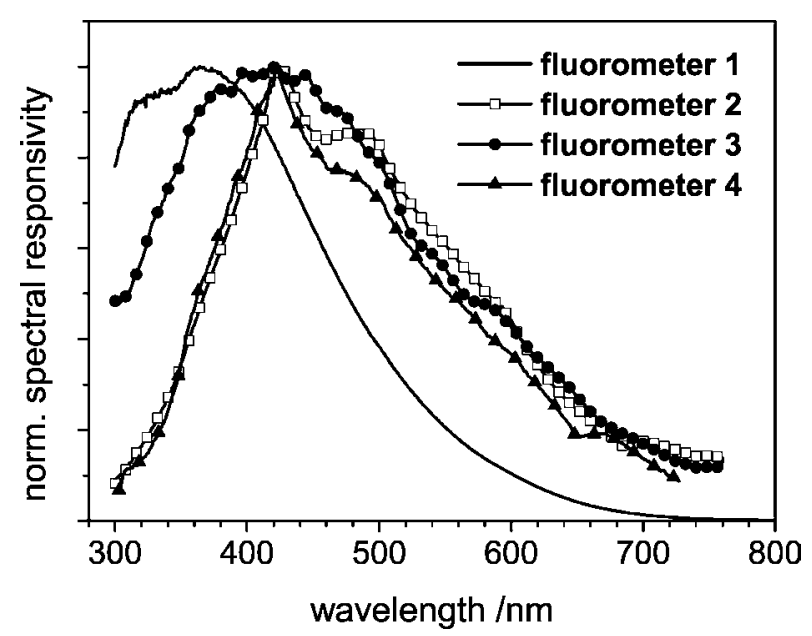

Fig. 1. Wavelength-dependent normalized spectral responsivity of the emission channel of four typical fluorometers equaling the corresponding normalized emission correction curves. Spectrally corrected emission spectra are obtained on division of the measured uncorrected spectra by the respective emission correction curve measured with identical instrument parameters. the respective instrument, with the size of these distortions depending on the spectral region of the dye's emission and the width of its emission band. Similarly, as has been illustrated in Parts I and II of a series of publications dedicated to the traceability in fluorometry $[40,42]$, the instrumentspecific wavelength and polarization dependence of the spectral irradiance at the sample position distorts excitation spectra and affects the intensity of emission spectra.

Other potential sources of error are uncertainties of the wavelength scale which need to be minimized especially for fluorophores with narrow absorption and/or fluorescence bands and nonlinearities of the detection system. The latter can hamper quantitative fluorometry and the determination of fluorescence quantum yields [40]. Furthermore, instrument- and sample-related polarization effects may play a role $[39,43]$. The size of instrument-related polarization effects is determined by the dependence of the transmittance and reflectance of the instrument's optical components on the polarization of the incident light and, to a much smaller extent, by polarization effects of detectors [40]. The magnitude of sample-related polarization effects depends on the fluorescence anisotropy of the sample. Instrument- and sample-inherent polarization effects, that are strongest for the measurement of anisotropic emitters without polarizers, can yield intensity and spectral errors in fluorescence measurements in the range of a few tens of percent [15]. Depending on the fluorescence technique, additional uncertainties can be introduced by e.g. an insufficient long-term instrument stability or instrument drift, an inhomogeneous illumination of the sample as well as generally by sample- and sample-handling-related effects. The latter are beyond the scope of this article.

As is often overlooked for certain analytes/samples, also type of light source, i.e. continuous or pulsed excitation, and related instrument settings such as e.g. the width of the time gate and delay have to be considered. This is illustrated in Fig. 2 for the normalized corrected emission spectra of a glass doped with rare-earth (RE) ions displaying different lifetimes in the $\mu \mathrm{s}$ - and ms-range that was measured with conventional steady-state fluorometers equipped with a continuous and a pulsed Xe lamp (width at half peak intensity of less than $10 \mu \mathrm{s}$ ), respectively. Such a RE-doped glass is an interesting candidate for a standard to control the wavelength accuracy and long-term stability of fluorescence instruments [44]. The differences observed in Fig. 2 are caused by the different and long lifetimes of the dopants and are not related to spectral correction. Basically, with the settings employed for pulsed excitation, suppression of the long-lived emission compared to the emission of the species with shorter lifetimes occurs. This observation correlates with the width of the time gate used for signal detection. Only 


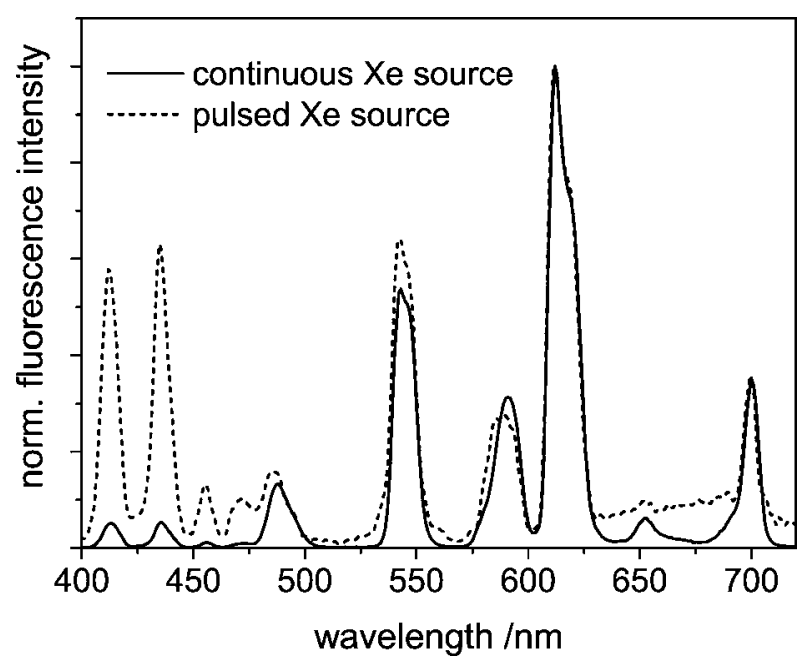

Fig. 2. Comparison of the corrected emission spectra of a glass, doped with a mixture of rare-earth (RE) ions, measured with a fluorometer equipped with a continuous excitation source (8100, Spectronics Instruments, Xe lamp, solid line) and a fluorometer with a pulsed lamp (LS 50B, Perkin Elmer, width at half peak intensity of the pulsed Xe lamp less than $10 \mu \mathrm{s}$, fluorescence mode, dashed line) using identical spectral bandpasses of the emission monochromators. Excitation was at $370 \mathrm{~nm}$.

minor effects are due to subtraction of dark current contributions and background from the long-lived emission. Accordingly, for instruments equipped with pulsed light sources, these experimental settings have to be considered for the comparability and reliability of fluorescence data if species with sufficiently long and different lifetimes are analyzed simultaneously. Furthermore, these parameters need to be kept constant for the control of the day-to-day performance and long-term stability of such fluorescence instruments.

Aside from instrument-specific effects, the reliability of fluorescence measurements as well as the limit of detection can be affected by non-specific or so-called background fluorescence. This includes all the fluorescence that does not originate from the fluorophore of interest. Background fluorescence may arise from contaminations of the sample and non-specific binding as well as from autofluorescence of optical components such as lenses, objectives, and optical filters [45] and sample supports/containers like glass or polymer slides and microtiter plates, respectively. The possible influence of autofluorescence from optical components is illustrated exemplary for filter glass OG 550 obtained by different pre-treatment procedures, see Fig. 3. Due to its clearly measurable autofluorescence on excitation at $450 \mathrm{~nm}$, the emissive filter is not suited for the fabrication of fluorescence instruments working in the UV/Vis region. These findings underline the importance of an improved quality control of such materials.

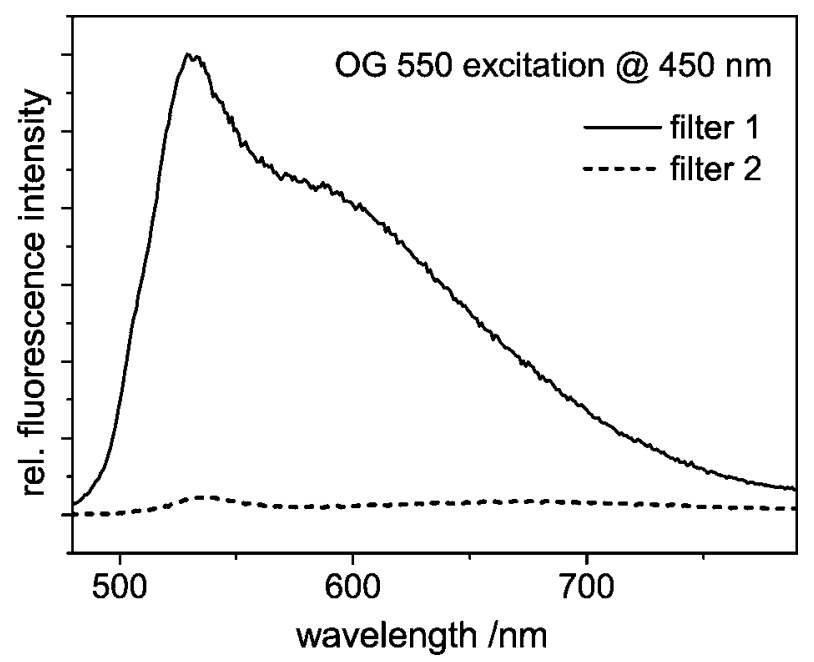

Fig. 3. Uncorrected emission spectra of two OG 550 filters obtained by different pre-treatment procedures recorded under identical conditions. Excitation was at $450 \mathrm{~nm}$.

\section{FLUORESCENCE STANDARDS: TYPES AND GENERAL REQUIREMENTS}

\section{Types of Fluorescence Standards}

The comparability of fluorescence data between instruments, laboratories, and over time as well as the minimization of fluorescence measurement-inherent sources of errors require a reliable instrument characterization and performance validation. This can be achieved with accordingly designed, well-characterized, and easy-touse fluorescence standards including standard operation procedures (SOPs) for their use. Additionally, as many fluorescence experiments are performed for quantification purposes, suited standards, recommendations for their choice and application as well as SOPs for the measurement of (relative) fluorescence intensities are desired.

Fluorescence standards are typically divided into instrument-type standards, which allow the determination of an adequate and application-relevant instrument performance thus ruling out instrumentation as a major source of variability, and more application-specific standards, which take into account the fluorescence properties of routinely measured samples. The latter are also referred to as reference standards for instance in fluorescence microscopy. Typical instrument standards are spectral standards with known, preferably certified corrected, i.e. instrument-independent fluorescence spectra. Spectral standards can be divided into wavelength standards to control the wavelength accuracy of wavelengthselecting optical components and so-called emission and excitation standards [14] for the determination of 
the spectral characteristics of fluorescence instruments [40,46-48]. Examples for more application-specific types of standards are fluorescence lifetime and fluorescence (de)polarization standards [16,49], that are beyond the scope of this article, and the class of fluorescence intensity standards. The latter include fluorescence quantum yield standards and reference materials to relate chemical concentration to instrument response or, less common, to characterize the autofluorescence of materials and reagents used for fluorescence analysis. Other equally requested standards, that belong to the classes of fluorescence intensity and spectral standards, are materials to control the day-to-day performance and the long-term stability of fluorescence instruments. At present, there is a broad variety of more or less suited fluorescence standards commercially available such as for instance fluorescent microspheres as alignment standards for fluorescence microscopy and flow cytometry, solid and liquid standards for the spectral correction of cuvette-type measurements and microplate readers as well as standards for front surface fluorescence measurements, and solid and liquid standards for intensity calibrations and for the determination of the linearity of microscopes, flow cytometers, and microplate readers, respectively [42,50]. In addition, there exists extensive literature on potential fluorescence standards. This includes for example emission and excitation standards and standards for fluorometric quantities like fluorescence quantum yield, fluorescence lifetime as well as fluorescence (de)polarization [1-4,14-15,51,52].

\section{General Requirements on Fluorescence Standards}

Generally, the requirements on fluorescence standards depend on the level of uncertainty desired for fluorescence measurements and accordingly, for instrument characterization. Standard-related contributions to the overall calibration and measurement uncertainty are directly linked to the reliability and suitability of the standard(s) and to the uncertainty of the standard's certified/reported radiometric/fluorometric quantities that are relevant for the respective application. For many applications, also the resemblance between the standard and typically measured samples affect the size of the standard-related contributions to the overall measurement uncertainty [40].

A purpose-fit fluorescence standard should enable the instrument characterization and IPV under analytically relevant routine conditions, e.g. for similar settings of instrument parameters (detector voltage, spectral bandpass, polarizer settings, signal intensities/counting rates, magnification etc.) and measurement geometry-preferably with a minimum and known uncertainty. This implies es- pecially comparable emission characteristics and spectral radiances or emission intensities of the standard and the sample, typically within a similar spectral region. The standard needs to be sufficiently well characterized with respect to its application-relevant spectroscopic properties. Its calibration-relevant radiometric/fluorescence features should have been determined with a reliably characterized reference instrument. To judge the reliability of the standard, the calibration procedure and calibration uncertainty of this reference instrument as well as the chosen instrument settings and measurement geometry need to be provided. Moreover, this eventually enables traceability to the spectral radiance scale and/or the spectral responsivity scale realized by the black body radiator and the cryogenic radiometer [40,42] and the mandatory report of the (wavelength-dependent) uncertainty of the respective fluorometric quantity of the standard. In addition, a specification of the standard's thermal and photochemical stability, the temperature dependence and-for some types of standards also the concentration dependence — of the reported/certified fluorometric quantity as well as information on storage conditions, shelf life, scope, and limitations for use are necessary [40]. For physical transfer standards, also recalibration intervals should be given. For chromophore-based reference materials, the purity and homogeneity of the chromophore and the solvent or matrix should be stated as well as the homogeneity of the dye distribution for solid systems. For particle-type standards, also the particle size distribution should be provided as well as the coloring procedure like for example staining, i.e. chromophore mainly inside the particle, or attachment to the particle surface, i.e. chromophore mainly at the particle surface and thus accessible to solvent.

\section{Type-Specific Requirements}

For each application, the choice of a fluorescence standard requires a thorough understanding of its purpose and, most important, the requirements it has to fulfill. A suited wavelength standard must emit a multitude of very narrow emission bands in the spectral range of interest, commonly the UV/Vis/NIR region, and must be measurable at sample position employing the typical measurement geometry. Furthermore, the spectral position of the emission lines/bands should preferably show no or at least a reported temperature dependence within the commonly encountered room temperature region. These demands are best met by a physical-type transfer standard, an atomic discharge lamp, ideally of cuvette-shape, typically in combination with a non-fluorescent reflectance or white standard [40]. Such lamps can be used for all types 
of luminescence instruments and are not restricted to photoluminescence measuring systems like chemical-type fluorescence standards. For reduced requirements on spectral resolution, also chromophore-based wavelength standards can be employed. Their advantages are the typically smaller spectral radiances and similar emission characteristics as encountered for the majority of samples. An example is a commercially available dysprosium-activated yttrium aluminum garnet $\mathrm{Y}_{3-x} \mathrm{Dy}_{x} \mathrm{Al}_{5} \mathrm{O}_{12}$ (DYAG, Photon Technology International Inc., FA-2036) that shows a multitude of narrow excitation bands in the spectral region between 250 and $500 \mathrm{~nm}$ and narrow emission bands in the spectral region from 500 to $800 \mathrm{~nm}$, respectively [53]. Similarly suited are mixtures of RE ions, see Fig. 2. Here, however, excitation wavelength-dependent emission spectra are encountered and accordingly, provision of the emission spectra for frequently used excitation wavelengths may be appropriate. Contrary to wavelength standards, the main requirement on emission and excitation standards that enable the determination of the relative spectral responsivity of the emission channel and the relative spectral irradiance at the sample position, respectively, are broad and unstructured fluorescence spectra to reduce the effect of instrumental resolution/spectral bandpass [40,47]. This minimizes the influence of uncertainties in the determination of the wavelength which are directly translated into uncertainties in fluorescence intensities. Further requirements are revealed in "Spectrally Resolved Macro- and Microfluorometry" section.

Fluorescence quantum yield standards are used as reference for the relative determination of the fluorescence quantum yield of an analyte. Accordinglycontrary to fluorescence intensity standards that relate chemical concentration to instrument response-they are typically not based on the same fluorophore as the sample and is not mandatory matching of spectra. The most stringent requirements are reliable fluorescence quantum yields and properly defined conditions such as e.g. matrix, oxygen concentration, temperature, and excitation wavelength as well as a stated uncertainty of the quantum yield. Furthermore, standard and sample should absorb and emit within comparable spectral regions, see also "Spectrally Resolved Macro- and Microfluorometry" section.

The working principle of the majority of fluorometric reference materials that relates chemical concentration to instrument response is the comparison of the spectral radiance/fluorescence intensity of a standard and a sample to quantify the number of fluorophores in the sample. Though often overlooked in fluorometry, this is a common approach used in many different analytical techniques.
This type of intensity standard typically relies on the same fluorophore(s) as to be quantified. However, due to the inherent sensitivity of the spectroscopic properties of the majority of chromophores to their microenvironment, this type of standard has to be handled with care. To eliminate errors in quantification, the chromophore to be specified and the standard have to be in the same microenvironment to guarantee identical fluorescence spectra, molar absorption coefficients, and fluorescence quantum yields, respectively. This is for instance fulfilled for many applications of high performance liquid chromatography (HPLC) with fluorescence detection where the fluorescence intensities from free, i.e. unbound fluorophores in solutions of identical or at least very similar composition are compared $[54,55]$. On the other hand, for a set of fluorophores in solution and a set of immobilized fluorophores, e.g. bound to or incorporated into a microparticle, the absorption and fluorescence spectra, molar absorption coefficients, and especially the fluorescence quantum yields can differ significantly [13]. If not properly considered, these differences lead to considerable errors in fluorophore quantification. As an approach to overcome these problems, the quantification of the fluorescence intensity of samples is often performed in units of molecules of equivalent soluble fluorochrome (MESF) [56,57]. This type of reference system, that circumvents differences in the molar absorption coefficients and fluorescence quantum yields of the standard and the sample, however, relies on matching fluorescence spectra and the sample and is not designed to derive the absolute number of fluorophores in the sample. Accordingly, standard-related contributions to the overall measurement uncertainty are linked to the degree of spectra matching of the standard and the sample [32]. This is also true for standards for the characterization of material-related autofluorescence which are requested by manufacturers of materials relevant for fluorescence analysis like e.g. optical glasses, crystals, and ceramics employed for the fabrication of optical components and supports/sample containers.

For standards employed for the characterization of the day-to-day performance and long-term stability of fluorescence instruments, a close match between the standard and routinely measured samples is not mandatory. However, these standards should be measured/measurable with routinely used instrument settings to guarantee the reliability of the instrument performance under applicationrelevant conditions. Further prerequisites are sufficient and reported stability or, for single-use standards, excellent reproducibility. Contrary to statements in the literature, per definition, there are no standards but only methods, procedures or guidelines-preferably in combination with suited materials - to measure the range of 
linearity of fluorescence instruments, their dynamic range and the limit of detection $[29,30]$. For instance, the determination of the former requires a method for the defined physical or chemical variation of the amount of light reaching the detector $[29,40]$. Additionally, recommendations on accordingly tested materials are desired that enable such measurements without material-specific contributions [40].

In addition to the standards discussed, for other fluorescence instruments like e.g. fluorescence microscopes, microarray scanners, and imaging systems, further reference materials are needed for instrument characterization and IPV. As is discussed in the sections dedicated to these techniques, requested are for instance standards for the determination of the spatial resolution, the correction for the heterogeneity of illumination and for detection within a single field, i.e. shading or flat field correction, and the characterization of the size of the illuminated volume.

\section{APPLICATION-SPECIFIC NEEDS FOR IMPROVED COMPARABILITY AND RELIABILITY OF FLUORESCENCE DATA}

Frequently used fluorescence techniques include macro- and microfluorometry often combined with measurements of fluorescence spectra, fluorescence lifetimes, and fluorescence (de)polarization, fluorescence microscopic techniques, and flow cytometry as well as fluorescence imaging in vivo. Fluorescence detection is also commonly employed for analytical separation techniques like for instance HPLC, capillary electrophoresis (CE), and (high performance) thin layer chromatography (HPTLC; TLC), and for bioanalytical methods like rt-PCR used for DNA analysis and DNA quantification as well as for the microarray technology. However-despite of the broad and ever increasing use of fluorescence techniques - to the best of our knowledge, there exist no overall accepted procedures for instrument characterization and IPV at present. Different manufacturers of steady-state fluorometers recommend the so-called Raman test [58,59] for the control of the instrument's long-term stability and sensitivity and the determination of the wavelength accuracy via scanning of the emission lines of the instrument's Xenon excitation source or the transmission minima in the spectrum of a solution of holmium perchlorate. These tests are often integrated into the software of instruments, and the recommended materials and required accessories are available from the instrument manufacturers, in some cases even with a SOP for use. More complex procedures recommended and commonly per- formed by service engineers include the determination of the wavelength accuracy with pen-type atomic discharge lamps and the characterization of the spectral responsivity of the emission channel with a calibrated lamp, i.e. typically an instrument-adapted integrating sphere-type spectral radiance transfer standard. The availability of such procedures is limited to few types of instruments, mostly steady-state fluorometers. For other techniques like e.g. fluorescence microscopy no such recommendations are available. Accordingly, in the following sections, the need for procedures/guidelines and standards for the characterization of fluorescence instruments and performance of fluorescence measurements is illustrated for selected fluorescence techniques.

\section{Spectrally Resolved Macro- and Microfluorometry}

\section{Uncertainty of Fluorescence Measurements}

The uncertainty [60] of fluorescence measurements relies on parameters such as e.g. the stability of the excitation light source and means for correction of its fluctuations, the stability and linearity of the detection system(s) and the detector's sensitivity to polarization effects, the occurrence of photobleaching, background fluorescence, scattering and reflections, the measurement geometry, sample format and reproducibility of sample positioning as well as the type of sample and sample-handling steps, respectively. Influences of the latter two factors are not further discussed. As a rule of thumb, for cuvette-type fluorescence measurements of transparent dilute solutions, the fluorescence-based uncertainty is $c a$. $2 \%$. For measurements of solid films with a routine fluorometer, the uncertainty is increased, mostly due to enhanced uncertainties related to sample positioning. For fluorescence measurements with 96-well microtiter plates, a well-towell reproducibility of $c a .2 \%$ can be achieved under optimum conditions, i.e. with well-aligned instruments, well-chosen microtiter plates, and a flat meniscus for top reading. The effect of the meniscus, that depends on the surface tension of the solvent, solvent-well interactions, and the volume of the analyte solution, is illustrated in Fig. 4 for top reading. Figure 4 compares the uncorrected mean emission spectra and the respective standard deviations (SD) obtained for two solutions of organic dyes, i.e. coumarin 153 and quinine sulfate which emit within the spectral region of $400-600 \mathrm{~nm}$ and were dissolved in ethanol and $0.1 \mathrm{M} \mathrm{HClO}_{4}$, respectively. For each measurement, the same black microtiter plate from NUNC and a volume of $270 \mu \mathrm{l}$ of the pipetted solution per well were used and the standard deviation was derived from 32 wells. The smaller SD of 3\% resulting for the ethanolic 


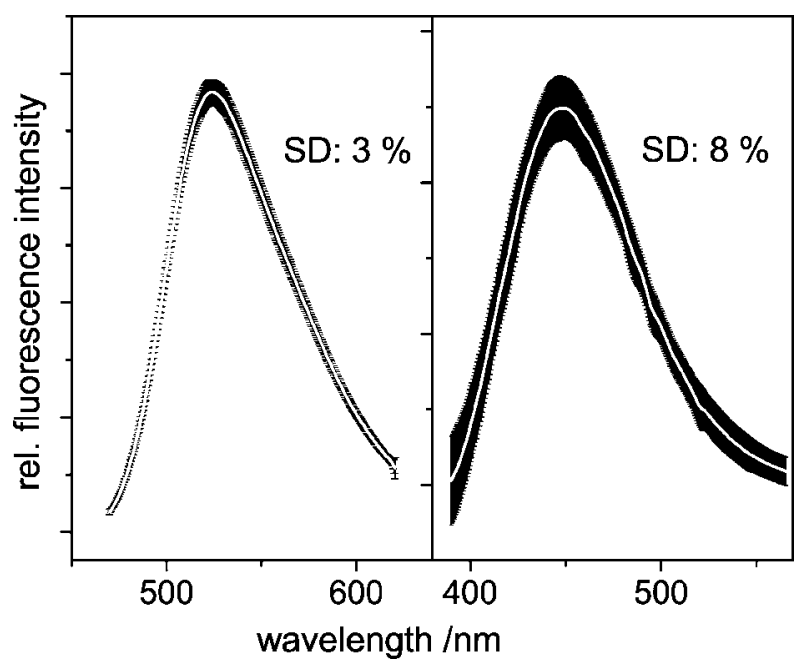

Fig. 4. Uncorrected mean emission spectra of two organic dyes and the respective standard deviations (SD) measured with a common microplate reader using a black 96-well microplate from NUNC and a pipetted volume of $270 \mu 1$, respectively. For each dye, spectra are averaged over 32 wells. Left: coumarin 153 in ethanol, right: quinine sulfate dihydrate in $0.1 \mathrm{M}$ perchloric acid.

coumarin 153 solution (left), which shows a rather flat meniscus, compared to the SD of $8 \%$ obtained for quinine sulfate dihydrate (right) exemplary demonstrates the influence of solvent-related meniscus effects.

\section{Need for Spectral Correction of Fluorescence Spectra}

The influence of the instrument-specific spectral characteristics and the according need for spectral correction is exemplary highlighted for steady-state fluorometry in Fig. 5 comparing the uncorrected and corrected emission spectra of a typical fluorescent label used for the derivatization of amino groups, here at the surface of a polymer support. The measurements were performed at two different settings of the emission polarizers, i.e. $0^{\circ}$ and $90^{\circ}$. As is evident from Fig. 5, the uncorrected emission spectra differ considerably in fluorescence intensity and shape for both polarizer settings. The bump in the uncorrected curve obtained for an emission polarizer set to $90^{\circ}$ arises from diffraction effects or so-called Wood anomalies of the monochromator gratings of the instrument. Moreover, both uncorrected spectra clearly deviate from the corresponding corrected spectra that are almost identical. These findings demonstrate the need for spectral correction to obtain comparable instrument-independent spectra and the potential of spectral correction to eliminate instrument-related polarization effects that can distort uncorrected spectra.

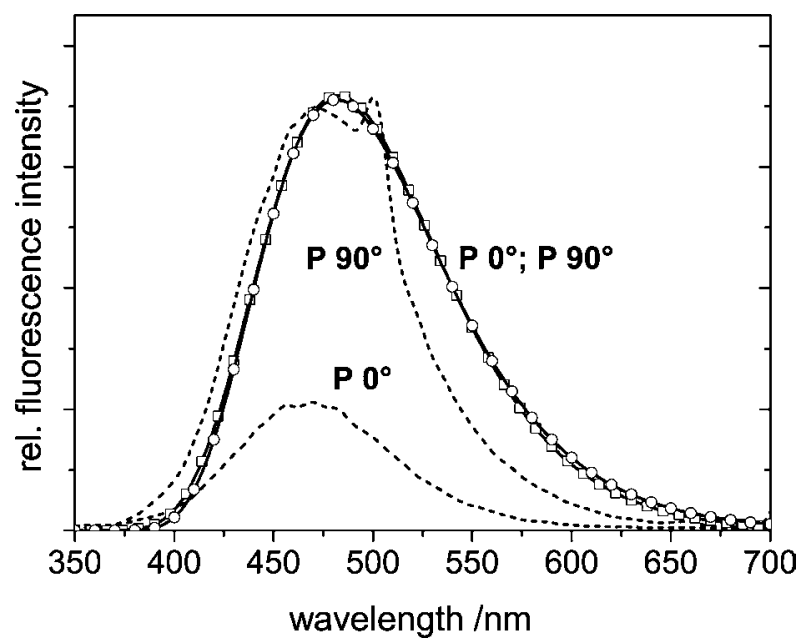

Fig. 5. Comparison of the uncorrected (dashed lines) and spectrally corrected (symbols) emission spectra of a fluorophore-labeled polymer film measured at two different settings of the emission polarizers, i.e. $0^{\circ}$ and $90^{\circ}$. The bump in the uncorrected spectrum obtained for $90^{\circ}$ results from diffraction effects of the monochromator gratings used.

\section{Fluorescence Standards Suited for Spectral Correction}

Spectral correction of fluorescence data provides the basis for the comparability of fluorescence data across instruments, laboratories, and over time as well as for the traceability of fluorescence measurements as illustrated in Figs. 1 and 5. With the increasing application of fluorescence instruments with real spectral resolution like for instance detectors for chromatography, that record fluorescence spectra, as well as modern types of fluorescence microscopes and the according desire to use fluorescence spectra for species identification, spectral correction, and the availability of reliably corrected fluorescence spectra are gaining importance for many other fields of application. Furthermore, spectral correction is mandatory for fluorescence methods that rely on the comparison of two fluorophores with different absorption and emission features or measured at two different wavelengths.

The traceable correction of fluorescence data for instrument-specific wavelength dependences can be realized via measurement of emission and excitation correction curves in relative intensities [40] either with physical transfer standards, i.e. calibrated standard lamps and detectors [42], or certified emission and excitation standards. Due to their ease of use and resemblance to typically measured samples, the latter approach is recommended for the broad community of users of fluorescence techniques. In addition to the previously discussed general requirements on fluorescence standards, and the need for broad and unstructured fluorescence spectra, this type of 
spectral fluorescence standard should have moderate to high fluorescence quantum yields independent of excitation or emission wavelength and dye concentration to enhance the signal-to-noise ratio and to reduce the influence of stray light, solvent emission, and fluorescent impurities on the shape of the standard's fluorescence spectrum [40,47]. Minimum overlap between absorption and emission is important to minimize inner filter effects. A nearly isotropic emission is the prerequisite to guarantee negligible additional uncertainties under measurement conditions that can dispense with or for instruments that lack polarizers. Furthermore, to cover the UV/Vis/NIR spectral region, sets of spectrally tailored standards are requested in combination with a tested procedure for the determination of the overall spectral correction curve from the measured and provided corrected spectra $[40,46,47]$.

\section{Measurement of Fluorescence Intensities}

To circumvent the use of standards, absolute measurements of fluorescence intensities or quantum yields can be performed. However, in addition to the consideration of the instrument's spectral characteristics, this requires the determination of the absolute fraction of incident photons per time unit in the sample that are absorbed by the analyte and the collection efficiency of the instrument, which together control the fraction of the fluorescence photons detected. This renders absolute measurements very challenging. Also, each instrument has to be accordingly characterized to establish the comparability of fluorescence intensities. On our opinion, this is not practicable on a broad level, i.e. for each instrument used for quantitative fluorometry.

For the broad community of users of fluorometry, a purpose-fit approach to quantitative fluorometry including the determination of fluorescence quantum yields is the application of fluorescence intensity standards. For these applications, the influence of spectra matching and spectral correction is exemplary illustrated in Fig. 6 for a series of commonly used fluorescent dyes, the corrected emission spectra of which are shown in the lower part of Fig. 6. The upper part of Fig. 6 depicts the integral fluorescence intensities of these dyes that have been determined relative to quinine sulfate dihydrate [61] taking the areas from the respective integrated uncorrected and corrected emission spectra, respectively. All the measurements were performed at the same excitation wavelength and bandpass. The emission spectra were backgroundcorrected and have been converted from radiometric to photonic quantities prior to integration [40]. As is evident

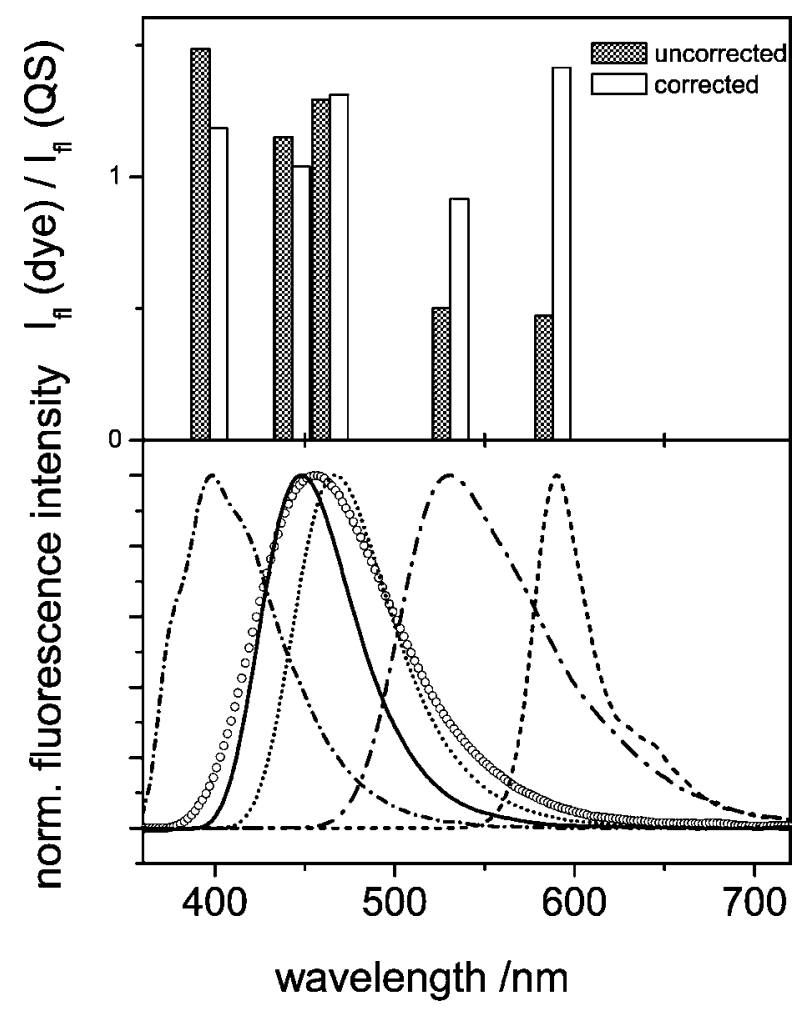

Fig. 6. Comparison of the integral fluorescence of several organic dyes obtained from uncorrected and corrected emission spectra, respectively, using quinine sulfate dihydrate in $1 \mathrm{~N}$ sulfuric acid as fluorescent standard. Excitation was at $370 \mathrm{~nm}$. Bottom: Corrected emission spectra of $\alpha$-NPO (short dash-dotted), coumarin 47 (solid line), coumarin 102 (dotted), coumarin 153 (dash-dotted), rhodamine 101 (dashed line), and quinine sulfate dihydrate (-o-).

from Fig. 6, the magnitude of these now and then considerable deviations between uncorrected and corrected data depend on the shape of the wavelength dependence of the spectral responsivity of the instrument used, see also Fig. 1, and for a given standard accordingly on the spectral region of the dye's emission and the width of its emission spectrum. For each sample-standard pair, uncorrected fluorescence spectra can be compared with a reasonable uncertainty only for species with identical or at least closely matching fluorescence spectra. This is the reason why for fluorescence techniques using integral measurements of relative fluorescence intensities for quantification purposes like e.g. flow cytometry, spectra matching is recommended $[32,56]$. Due to the instrument specifity of spectra matching of a sample-standard pair, its dependence on dye microenvironment, and the ever increasing variety of fluorescent labels and fluorophorecontaining systems, general solutions to quantitative fluorescence analysis with small uncertainty are not very 
realistic. Fluorescence-based uncertainties of $c a .2 \%$ can be realized only for simple cases where the fluorescent analyte and the standard are identical and located in the same or in a very similar microenvironment. This is often met e.g. for HPLC analysis of fluorescent analytes such as polycyclic aromatic hydrocarbons $(\mathrm{PAH})[54,55]$. However, for more complex systems, a considerable increase in measurement uncertainty of up to a few tens of percent may occur. This is further detoriated especially by the dependence of the fluorescence quantum yield on microenvironment. To meet this challenge, it may be of interest to determine fluorescence-based uncertainties for representative examples, i.e. very popular labels and matrices, with varying degree of spectra matching and well-characterized microenvironment-induced changes in molar absorption coefficients and fluorescence quantum yields.

\section{Fluorescence Quantum Yield Standards}

From the material side, the sensitivity of fluorescence analysis depends on the molar absorption coefficient of the sample/analyte(s) at the excitation wavelength and the fluorescence quantum yield. Accordingly, the availability of fluorescence quantum yields of (bio)analytically relevant dyes in application-relevant matrices under applicationrelevant conditions and their accurate determination are of considerable importance. The uncertainty of the measurement of relative fluorescence quantum yields is governed by several factors. Major contributions arise from the uncertainty or lack of spectral correction, see Fig. 6, temperature-related effects, uncertainties inherent to absorption measurements and dilution of solutions as well as from the uncertainty of the fluorescence quantum yield of the chosen standard, respectively. With respect to the latter, it is critical that literature data on fluorescence quantum yields are often not very reliable with deviations of up to $50 \%$ being not uncommon and uncertainties typically missing. Accordingly, there is still a considerable need for fluorescence quantum yield standards for the UV/Vis/NIR spectral region, the fluorescence quantum yields of which have been determined with a stated uncertainty with a traceably characterized setup. To eventually overcome these problems, currently at BAM, a reference fluorometer is been built for the dissemination of absolute fluorescence quantum yields. Furthermore, not only well-characterized standards with high fluorescence quantum yields are desired but also systems of low fluorescence quantum yield. This takes into account that not only the absorption and emission spectra of standard and sample should lay within a similar spectral region but also the size of their quantum yields should be preferably close to avoid problems related to nonlinearities of the detection system or dilution errors.

\section{Standards for the Characterization of Material-Related Autofluorescence}

The inherent sensitivity of fluorescence imposes strong requirements on materials suited for the fabrication of optical components for fluorescence instruments as well as supports for fluorescence analysis. This eventually affects quality control by materials suppliers as is exemplary revealed for glass-type materials. Generally, materials made for applications in optical technologies should show minimum autofluorescence on excitation at wavelengths typically used, i.e. LED, and laser (diode) wavelengths as well as at 365,248 , and $193 \mathrm{~nm}$. Furthermore, they need to be stable under application-relevant conditions, e.g. at photon fluxes in the range between $\mu \mathrm{W} / \mathrm{cm}^{2}$ for spectroscopy/microscopy and $\mathrm{kW} / \mathrm{cm}^{2}$ for microlithography, and should not form fluorescent photoproducts $[62,63]$. With respect to proper quality control for glass-type materials, it has to be taken into account that their optical properties and (photo)stability are influenced by (fluorescent) impurities/dopants, the presence of point defects such as color centers that originate for instance from impurities such as RE elements, as well as the matrix. Also, the glass manufacturing process itself plays a role, i.e. redox equilibria and eventually incorporation of dopants into the glass matrix [64-66]. Accordingly, material-related autofluorescence needs to be specified under application-relevant conditions, e.g. excitation wavelengths and photon fluxes [67], and on a batch-to-batch basis, see also Fig. 3. Techniques that are increasingly used for this purpose are conventional steadystate, laser-induced, and time-resolved fluorometry [6874]. For instance, a standard procedure for quality control used by suppliers of optical glass is the measurement of the glass emission at an excitation wavelength of $365 \mathrm{~nm}$ and comparison of the integral emission in the wavelength region of 400-700 nm to that of reference samples like SF1 or SF6 available from Schott AG. SF1 and SF6 contain a defined amount of emissive lead ion chromophores, e.g. ranging between 20 and $70 \%$. A similar approach based on lead-type glasses certified by the Agency of Industrial Science and Technology (AIST) is performed within the Japanese Optical Glass Industrial Standard (JOGIS) procedure applied by Japanese manufacturers [75]. Glasses of low emission show an integral photoluminescence well below $5 \%$.

Despite of the considerable importance for optical industry, instrument manufacturers and for the reliability of fluorescence analysis-aside from these lead-based 
standards - up to now, there are no suited solid reference materials available that enable a standardized determination of the application-relevant fluorescence properties of glass-type materials. Desired are fluorescence quantum yield standards and/or reference materials that relate the concentration of typically encountered fluorescent impurities or dopants to fluorescence intensity, standards to quantify the acceptable level of autofluorescence for quality control as well as standards for the fluorometric quantities luminescence lifetime and (de)polarization. In addition to the requirements on quantum yield and fluorescence intensity standards, of special importance for this application are the shape and size of potential reference materials and the respective measurement geometry, e.g. $0^{\circ} / 90^{\circ}$ and front face. Also the luminescence quantum yields of the analytes of interest have to be taken into account. Luminescence quantum yields are in the range of $60 \%$ for RE-doped optical glass and $\mathrm{CaF}_{2}$ due to the inner-shell nature of the optical transitions involved, reach up to $95 \%$ for color centers [76], and are below $10 \%$ for optical transitions from the $\mathrm{ns}^{2}$ levels $(n=1,2,3$, etc.) as is found for instance in $\mathrm{SbO}_{3}, \mathrm{AsO}_{3}$ or $\mathrm{ZnO}$. Suited luminescence lifetime standards should cover the ns- to ms-time domain. In a first step, to help manufacturers of optical materials and fluorescence instruments to improve fluorescence-based quality control, a set of reference materials covering the most important application-relevant problems related to autofluorescence from glass-type materials needs to be defined, certified, and made available. Furthermore, internationally accepted guidelines for the determination and specification of autofluorescence are increasingly requested. This will eventually contribute to an improved performance of fluorescence instruments and reliability of fluorescence analysis.

\section{Flow Cytometry}

Flow cytometry is a technique frequently used in clinical medicine to support diagnoses or prognoses of different diseases such as leukaemia, HIV infection or immune paralysis, i.e. sepsis. To derive the information required for a medical decision, cells of a blood or bone marrow sample as well as cells isolated from a biopsy are distinguished according to their physical and biological properties. Subsequently, concentrations or relative concentrations of subpopulations of cells are determined. Apart from cell differentiation based on light scattering in forward and sideward directions, staining of the surface of target cells by fluorophore-labeled monoclonal antibodies has evolved as a powerful tool for the identification of subpopulations of cells, e.g. T-helper and T-suppressor lymphocytes in AIDS diagnosis. In addition, flow cytom- etry is used to detect fluorescently labeled intracellular constituents like DNA or messenger RNA.

One essential feature of flow cytometry is the analysis of single particles at a high throughput of up to $100 \mathrm{kHz}$. For this purpose, the sample fluid containing the suspended particles is injected into a flow cell together with a second fluidic stream, the sheath or mantle stream. This results in hydrodynamic focusing of the sample stream to diameters of typically $5 \mu \mathrm{m}$ that correspond approximately to the size of human blood cells. Hence, the cells are forced to cross one or several laser beams, oriented perpendicularly with respect to the direction of the flow, in single file. When passing the laser beams, for each cell, light scatter in forward and sideward direction and laser-induced fluorescence is simultaneously recorded. Generally, the pulse heights of the signals are measured. Fluorescence is observed in up to four different optical bandpasses or channels corresponding to different antibodies and different fluorophores, respectively.

In most cases, relative fluorescence intensities are measured in flow cytometry to divide cell population into two species, one of which reacts with a selected monoclonal antibody and hence is termed positive. The other population that does not react with the selected antibody is referred to as negative. In Fig. 7, a typical histogram obtained with the antibody CD4 (Cluster of Differentiation 4) labeled with the fluorophore PE (phycoerythrin) is presented which features two populations, i.e. CD4positive $\left(\mathrm{CD} 4^{+}\right)$lymphocytes and CD4-negative lymphocytes [77]. The difference or ratio of the distributions of fluorescence intensities between these two cell subsets strongly depends on several factors. This includes the instrumental characteristics, the spectroscopic properties

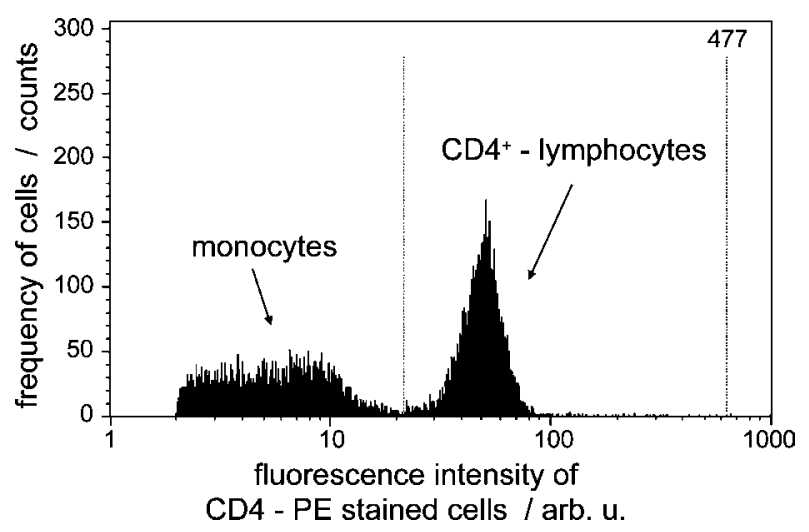

Fig. 7. Histogram of the frequency distribution of stained white blood cells. Phycoerythrin (PE) labeled antibodies CD4 were used to identify CD4-positive T-lymphocytes. Included in the figure is the discrimination threshold to distinguish positive and negative cells. The histogram was provided by V. Ost, PARTEC GmbH. 
of the fluorescent molecules attached to the antibody, and cell-specific biological features like autofluorescence from intracellular constituents and non-specific binding. To account for contributions arising for example from autofluorescence and from non-specific binding, control experiments (variation of the spectral irradiance of exciting laser beams, isotype control to determine non-specific binding, titration of antibody concentration) are required to optimize the difference between positive and negative populations, i.e. the signal-to-background ratio. To facilitate the comparison of the results from different instruments, to compare different blood samples, and to accomplish a day-to-day control of the flow cytometer, stained microbeads are frequently used. A typical histogram displaying the relative frequency of particles of different fluorescence intensity is depicted in Fig. 8. The number at each peak corresponds to MESF units. The MESF number does not represent the true amount of dye molecules, but indicates the brightness, i.e. the product of the molar absorption coefficient and the fluorescence quantum yield, of the fluorescent species in solution [13,39]. MESF particles are available with different fluorophores like for instance fluorescein (FITC), PE or allophycocyanin (APC) and are routinely used to align the optics of flow cytometers, to set the detection threshold as well as to check the sensitivity and the linearity of the instruments. Alternatively, the linearity and resolution, i.e. the width of the intensity distribution of flow cytometers can be monitored with biological material, e.g. DNA-stained trout or chicken erythrocytes.

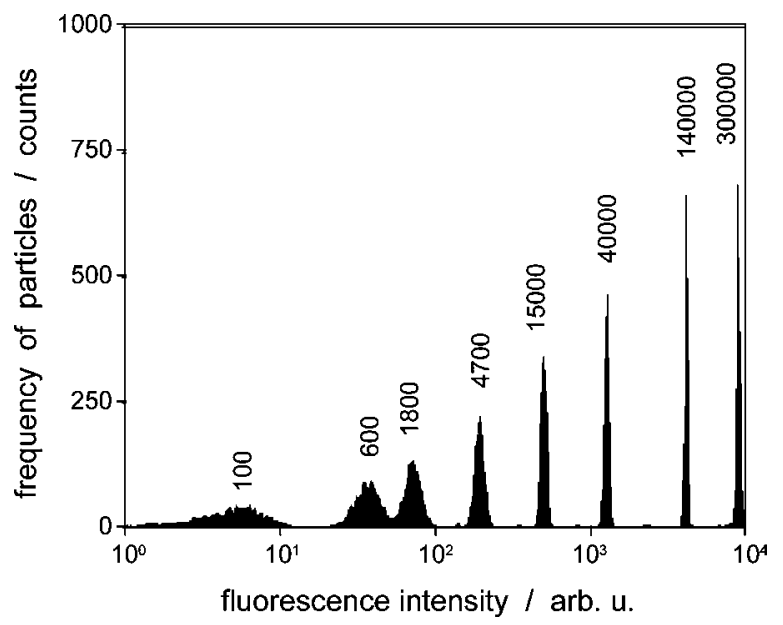

Fig. 8. Histogram of differently stained calibrations beads (SPHERO ${ }^{\mathrm{TM}}$ Rainbow Fluorescent Particles). The fluorescence intensities given in arbitrary units can be calibrated in MESF units. The corresponding MESF numbers labeling each peak indicate its respective center. The fluorescence was observed in the FITC detection path of a Cytomation MoFlo ${ }^{\mathrm{TM}}$ cell sorter.
To improve quality assurance in flow cytometry, the spectroscopic features of existing calibration (MESF) particles should be better characterized. This implies for example the determination of the absorption spectrum and the molar absorption coefficients at the wavelengths commonly available in flow cytometers as well as the corrected emission spectrum and fluorescence quantum yield in phosphate buffered isotonic solution. The availability of such data would allow users of flow cytometry to obtain a spectral correction of the instrument and to determine the crosstalk between different fluorescence detection paths, the correction of which is termed fluorescence compensation. This eventually considers future developments in flow cytometry that might result in an improved spectral resolution from presently 20-30 to $1-2 \mathrm{~nm}$. Accordingly certified MESF beads are suited to calibrate such instruments which allow to record emission spectra.

Apart from relative measurements, quantification of fluorescence intensity in flow cytometry and subsequent deduction of the significant biological quantities, i.e. the number of specific antibodies on the cell surface (antibody binding capacity, $\mathrm{ABC}$ ) or the number of proteins within the cell, would possibly allow to improve medical diagnosis. For example, standardized immune monitoring, which includes the determination of $\mathrm{ABC}$ for different antibodies, has been recently applied [78] to derive criteria for the prediction of infections in risk patients after cardiopulmonary bypass surgery. For monocytes, the expression of HLA-DR (human leukocyte antigen) epitopes correlates with their activation and can be applied to monitor patients with septic shock [79]. To determine the number of antibodies, the QuantiBRITE ${ }^{\mathrm{TM}}$ principle is used [80]. For this calibration procedure, PE is chosen to label antibodies and calibration beads as the influence of the microenvironment on its spectroscopic properties is not as pronounced as for other fluorophores. Another advantage of PE is its high molecular weight and its correspondingly large size, which allows to control that only one fluorochrome is bound to each antibody. Apart from quantification of PE fluorescence, the expression of enhanced green fluorescent protein (EGFP) of transfected T-cells has been determined by calibration of the fluorescence of cells to an EGFP standard of validated MESF units [81].

Due to the inherent sensitivity of fluorescence to dye microenvironment, for each chromophore, diverse environmental conditions can cause significant variations in the brightness and spectral position of the absorption and emission bands of the stained cells. The classical example is the $\mathrm{pH}$ dependence of the fluorescence quantum yield of frequently used fluorescein which limits its use. Hence, to correct for such variations, any standard used to derive 
biological quantities has to mimic the fluorescently labeled cells as closely as possible. However, because of the complexity of bacteria or cells, it is challenging to achieve matching of absorption and emission spectra of standard and biological sample. In addition, it is ambiguous to control labeling-induced changes of the spectral features, the molar absorption coefficient, and especially the fluorescence quantum yield. To assure the traceability of such a standard and to quantify its spectral properties, comparison with corresponding stained cells is mandatory, for example by employing cell sorting and subsequent single molecule bleaching under microscopic control. This technique would allow to determine the properties of fluorophores in situ and to account for the influence of the microenvironment. In addition, to derive the biological influencing quantities, colocalization should be applied to the cells under investigation to distinguish specific and nonspecific binding of the fluorophore-labeled monoclonal antibodies.

\section{Fluorescence Microscopy}

As a tool in microscopy-in addition to absorption methods-fluorescence provides a number of already mentioned possibilities such as e.g. selectivity of communication via different parameters like excitation and emission wavelength, fluorescence lifetime, and fluorescence (de)polarization [5,82-85]. Furthermore, with confocal laser scanning microscopy and wide-field microscopy with spatially modulated excitation, the theoretical limits of spatial resolution that are determined by the numerical aperture of the objective and the excitation wavelength can be realized in practice. In addition, labeling or probing of biological structures with fluorescent reporters allow their indirect visualization even when the size is far below the optical resolution limit. Accordingly, confocal and wide-field fluorescence microscopy have been developing into some of the most powerful and commonly used methods in medicine, forensics, and bioanalysis. Founded on these techniques, further specialized imaging and non-imaging methods like two-photon fluorescence microscopy (2P), Förster or fluorescence resonance energy transfer (FRET), total internal reflection microscopy (TIRF), fluorescence lifetime imaging microscopy (FLIM), fluorescence recovery after photobleaching (FRAP), fluorescence loss in photobleaching (FLIP), fluorescence intensity (FLINT), fluorescence anisotropy (FA), time-resolved energy transfer (TRET) as well as fluorescence correlation spectroscopy (FCS) have been become very popular. Typical applications of fluorescence microscopy techniques are investigations of fixed (dead) samples like immunofluorescence studies and in situ hybridization measurements of DNA sequences, live cell imaging with measurements of the structure, dynamics, and potential (maps) of membranes and organelles, measurements of cell structures, organization, and function, e.g. determination of intracellular $\mathrm{pH}$ and physiologically important ions like e.g. $\mathrm{Ca}(\mathrm{II}), \mathrm{Na}(\mathrm{I}), \mathrm{K}(\mathrm{I})$, and $\mathrm{Mg}(\mathrm{II})$ [86] as well as studies of protein structure and dynamics $[5,82-84,87]$. A broad variety of these microscopic techniques do not require quantification. However, methods for the study of biological functions are gaining importance that demand the precise quantification of the concentration of analytes by measuring fluorescence intensity in the 3D space over time (4D). Furthermore, as some experimental approaches or methods like FRET require quantitative spectrally resolved measurements of two fluorophores simultaneously, this task can result in $5 \mathrm{D}$ data sets. The need for quantification, however, is poorly met at present as commercial imaging instruments are designed mostly for high image quality, i.e. low background signals and image distortions, high light throughput, and good detection efficiency, but not primarily for quantitative measurement of fluorescence. Accordingly, this situation is reflected by the commercially available standards.

Due to the complexity of the instrumentation used for confocal and high-end wide-field imaging microscopy, there is an urgent need for easy-to-use standards and simple procedures for the characterization of these instruments, control of instrument specification, day-today and long-term performance as well as to enable the comparability - and in some cases the combination of microscopic data—across instruments and laboratories. This can be seen as the first necessary step towards calibration procedures and standards fulfilling the up-coming need for signal quantification in many fields of microscopic imaging. The demand for standardization of instrument characterization and measurement procedures is further enhanced due to the increasing use of fluorescence microscopy in areas like medical diagnostics. Accordingly, in this section, the general need for and requirements on standards suited for fluorescence microscopy are discussed without consideration of the specific demands of the broad variety of fluorescence microscopic techniques available.

In conventional and confocal fluorescence microscopy, the recorded signal is affected by the samplespecific amount of fluorescent molecules with a certain molar absorption coefficient and fluorescence quantum yield present in a given microscopic volume, the instrument, and autofluorescence. The latter can arise for instance from the sample, the instrument's optics and/or from an immersion medium. (Time-dependent) 
instrument-specific effects are linked to the wavelengthdependent spectral irradiance at sample position, the wavelength- and polarization-dependent light collection properties and aberration of the microscope, and the wavelength- and polarization-dependent spectral responsivity of the detection system [88]. To obtain instrumentindependent and hence comparable data, these instrument characteristics need to be measured. For the quantification of the recorded signals, the linearity of the detection system has to be determined and either standards closely matching the sample/analyte to be quantified or appropriate characterization procedures are required [89]. According to the broad community of fluorescence microscopists [90], instrument-type standards - preferably in combination with guidelines for instrument characterization-are desired to characterize the size of the illuminated volume, i.e. the point-spread function [91], the instrument's spatial $(x, y)$ resolution, the spectral irradiance/excitation intensity reaching the sample, the homogeneity of the sample illumination, the field flatness, $z$-distance and $z$-resolution, the spectral resolution as well as the day-to-day and long-term instrument performance, respectively. Moreover, application-specific fluorescence intensity standards or so-called reference standards are requested [92] and internationally agreed methods/protocols in combination with suited systems to determine the range of linearity of the detection system [93]. Additionally, guidelines including materials to measure limits of detection are of interest, for instance to compare the sensitivities of different instruments. Furthermore, with the increasing spectral resolution offered now by the majority of modern commercial confocal microscopes, spectral fluorescence standards are gaining importance [94].

Standards for the characterization and calibration of fluorescence microscopes have to meet additional requirements compared to standards for macroscopic fluorescence spectroscopy. This is due to the strongly increased spectral irradiances at the sample position that exceed those used in conventional (macro)fluorometry often by 4 or more orders of magnitude [17]. Accordingly, frequently enhanced photodecomposition is observed, especially in the case of laser illumination typically used for confocal scanning techniques. The strongly improved spatial resolution imposes more strict requirements on the homogeneity of the chromophore distribution within the standard. Size and shape of a standard are by far more important as microscopic measurements usually require changes in field aperture and magnification. Only dimension-adapted microscopic standards with a well-defined shape permit a correct intensity/volume relationship. Furthermore, the spectral radiances and spectral characteristics of the standard and the sample need to be sufficiently similar.
At present, there is a broad variety of fluorescence standards, mostly of instrument-type, commercially available. Instrument standards can be of chemical, i.e. organic or inorganic nature, or of physical type. Standards, which relate instrument response to analyte concentration, commonly rely on organic fluorophores either incorporated within or bound to the surface of a stable, non-fluorescing host of well-defined shape. Other approaches are microdroplets of fluorescent solutions [92] or microcapillaries filled with fluorophore solutions $[17,95,96]$. Typical instrument standards are for instance inorganic ion-doped glass materials such as uranyl-containing glasses $[97,98]$ that are of good photo-stability but no longer commercially available, or inorganic ion-doped fibers [99] developed for the calibration of the day-to-day instrument performance. Inorganic ion-doped glasses have only restricted application as reference standards for certain analytes with sufficiently similar excitation and emission spectra. Fluorescent microspheres [100-102] are of widespread use not only as instrument-type standards to check on instrument alignment, sensitivity, and stability as well as spectral separation (multicolored particles) of both conventional microscopes and laser scanning systems but also as internal as well as external fluorescence intensity standards. Some examples for measurements with fluorescent microspheres are shown in Fig. 9. The dually labeled beads (FocalCheck, Molecular Probes) shown are applied for alignment purposes (parfocality, xy pixel-shift with color) in confocal and wide-field fluorescence microscopy when two or more excitation/fluorescence channels have to be acquired from one location in the 3D space. They allow to adjust for pixel-shift free recordings in the 3D space, see Fig. 9C and D, respectively. However, these microspheres can act as an additional optical lens element in the optical path, leading to severe optical distortions in the 3D space as follows from Fig. 9B. The applicability of such particles is further limited by the fluorophore's stability and often by the mounting medium.

Other suggested standards include concentrated solutions of fluorophores like fluorescein and rhodamine dyes on a regular slide [103] for shading or flat field correction, fixed fluorescent cells [104] or fluorescent polymers, which can, however, suffer from spatial non-uniformity leading to inhomogeneous emission [105], immobilized particle arrays [106] or wax films doped with fluorescent dyes [107]. Recently, organic and inorganic systems containing uniformly dispersed luminescent nanocrystals at various concentrations have been suggested as potential fluorescence standards for microscopy [108]. However, aside from the generally advantageous spectroscopic properties of quantum dots, i.e. size-tunable broad absorption and narrow emission bands, and comparatively high 

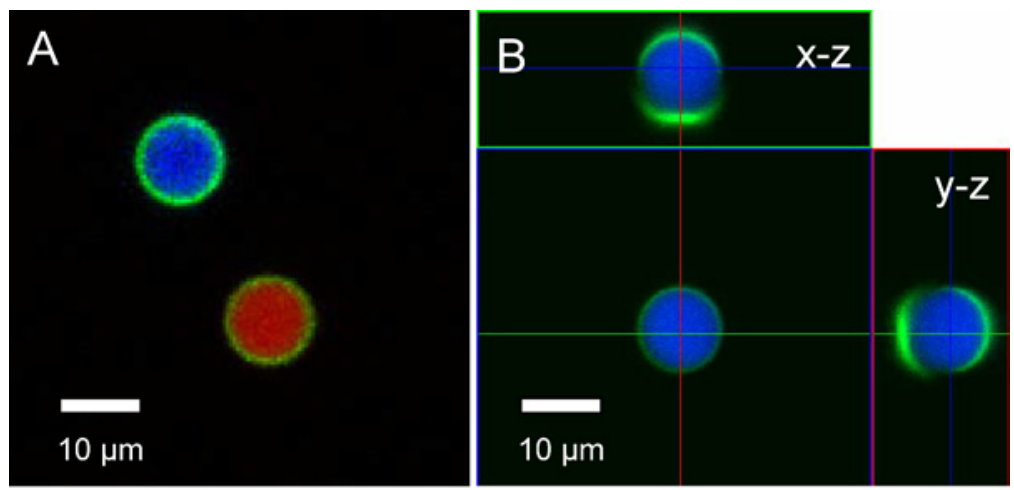

$10 \mu \mathrm{m}$
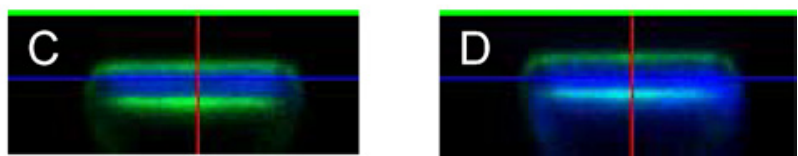

Fig. 9. Focal check beads usable for parfocal alignment and shift free multi-channel acquisition in confocal and wide-field fluorescence microscopes. The beads are labeled with one color throughout and with a second color on the outer shell. (A) Overlay of a three-channel image acquired sequentially with 364,488 , and $543 \mathrm{~nm}$ excitation (mixture of beads F-7237 and F-7238, Molecular Probes Inc., Eugene, USA). (B) Orthogonal $x-z$ and $y-z$ cross-section view from a two-channel $z$-stack (excitation at 364 and $488 \mathrm{~nm}$ ). It can be seen, that the bead acts as an optical active element, leading to a distorted intensity profile of the green colored shell. (C) $x-z$ cross-section view of a bead squeezed between two microscope slides, well-aligned collimator for UV/Vis illumination. (D) $x-z$ crosssection after misaligning the collimator, which results in a shift of one color in relation to the other. All images were recorded with a $63 \times / 1.2$ water immersion lens on a LSM 510 Meta confocal microscope (Carl Zeiss GmbH, Jena, Germany).

fluorescence quantum yields in the long wavelength region [109], the suitability of such systems as standards requires a very narrow and reproducible particle size distribution and a time- and illumination-independent brightness [110]. The latter implies the overcome of the often observed increase in fluorescence intensity on illumination or so-called photobrightening [111]. Also physical-type standards like for instance lamp-based calibration assemblies have been reported [94]. An intriguing approach is a test slide that contains a built-in regulated light-emitting diode in feedback configuration with a PIN photodiode and different pinholes to mimic the emission characteristics of fluorescent cells [112]. This assembly allows the determination of the linearity of the detection system of a microscope. Another multifunctional standard is a microscope test slide that contains side by side a photodiode for power and pulse length measurements and a detector for wavelength measurements [113].

Despite of the enormous progress in fluorescence microscopy and the broad variety of fluorescence standards suggested, up to now, the suitability of the available materials as well as the need for improved standards for the characterization of fluorescence microscopes and for quantification purposes is still under debate [90]. This is also true for the demand of internationally accepted protocols for instrument calibration, control of instrument specifications, and IPV as well as signal quantification. Disadvantages of some of the available or recommended fluorescence standards that need to be considered and eventually overcome, are high, not sample comparable fluorescence intensities emitted even at low excitation intensities which lead to enhanced calibration uncertainties caused by nonlinearities of the detection system or stray light effects within the instrument. Critical can be also a non-adequate thickness of the fluorescence standard that renders the identification of the appropriate focal plane for the calibration procedure difficult. Also, the effect of the coverslip has to be taken into account for the design and selection of a suited standard. Measurements of a standard without a coverslip may be erroneous due to distortion of the measured signal in the light path, as most of the objectives for biological imaging are designed, i.e. calculated, for use with a coverslip of $170 \mu \mathrm{m}$ thickness. However, for objectives made for application without a coverslip-water dipping lenses employed in neurobiology or lenses for use in material science-coverslip-free standards are needed. Furthermore, severe optical distortions due to strong refractive 
index mismatches between standard and immersion media can be problematic and should be avoided when the 3D dimension of the standard is of importance. Other standard-related problems and sources of error can be an insufficient or not reported stability under laser illumination that leads to photodecomposition of the fluorophore and accordingly aging-inherent intensity and/or spectral variations and/or an inhomogeneous distribution of the chromophore within the matrix for solid standards such as color slides.
To the best of our knowledge, long-term stability problems have been encountered for almost all types of potential standards for fluorescence microscopy. The influence of such photoinduced effects is exemplary illustrated in Fig. 10 for colored, fluorescent plastic slides that are used in confocal and wide-field microscopy for spectrally resolved measurements, spectral calibration as well as adjustment or control of the homogeneity of the illumination. These slides may be also suited for the evaluation of (fluctuations of) the excitation light intensity or
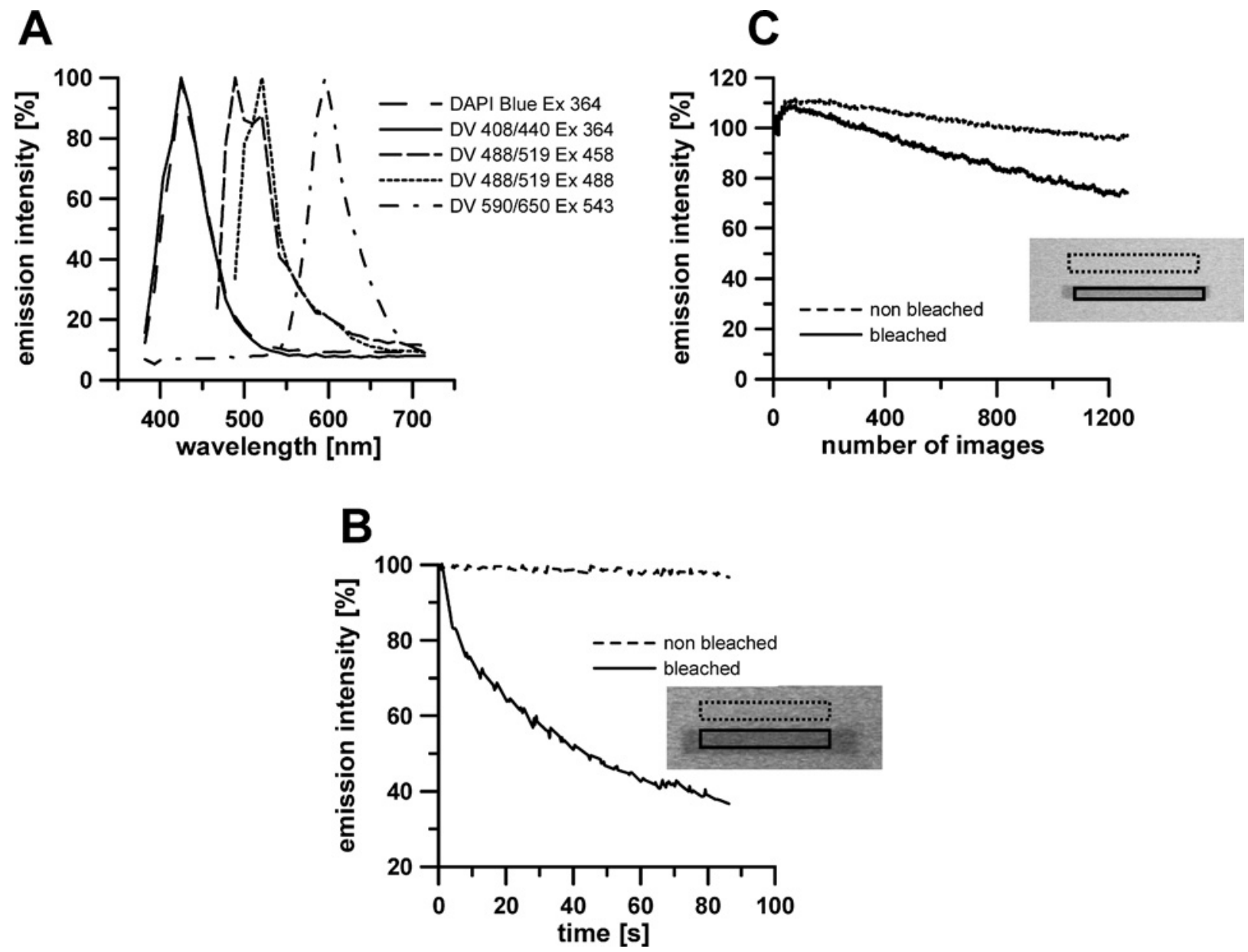

Fig. 10. Fluorescence emission spectra and photobleaching of plastic color microscope slides from different suppliers (Deltavision (DV), MM). (A) Emission spectra of the color slides recorded on a LSM 510 Meta (Carl Zeiss GmbH, Jena, Germany) confocal microscope with the appropriate primary dichroic beamsplitter (DC) and a $63 \times / 1.2$ water immersion lens. The bandwidth of the spectral Meta unit was set to $10.2 \mathrm{~nm}$. A distortion of the real emission spectrum as a result of the optical properties of the DC was not taken into account. (B and C) Photo-stability/bleach resistance of the calibration slides. The measurements of times series of images were performed as follows: Within regions of interest (ROI), marked with solid lines, additional scans with maximum laser intensity were run to probe the bleaching characteristics of the slides. The settings for these ROI were: $1000 \mathrm{scan}$ iterations, pixel time $1.4 \mu \mathrm{s}$, scanning time in total for the ROI ca. $2.5 \mathrm{~s}$ (DAPI Blue, Ex $364 \mathrm{~nm}$,) or $25 \mathrm{~s}$ (DV $488 / 519$, excitation at $488 \mathrm{~nm}$ ). This was repeated with the DAPI Blue sample (B) every $2.5 \mathrm{~s}$ for 20 times and for DV 488/519 (C) every $2.5 \mathrm{~s}$ for 60 times. Images of the total area shown were taken in between the high-intensity ROI excitation: Five images (DAPI Blue) and 10 images (DV 488/519). Measurements of the emission intensity over time within the upper ROI positions result in the non-bleached curves (dashed lines), the ROIs marked with solid lines yield the bleached curves. 
the determination of the sensitivity of fluorescence microscopes. The emission spectra of these slides and the photostability studies are shown in Fig. 10A, 10B, and 10C, respectively. The low resistance to photodecomposition in combination with the presence of defects in the fluorescent layer makes these slides not very attractive candidates for other than spectral calibration tasks provided that no lightinduced spectral changes occur. To overcome problems related to photodecomposition requires either materials of an improved or at least better characterized photo-stability or single-use materials of excellent reproducibility.

A first step to the design of better-suited fluorescence standards for fluorescence microscopy seems to be the development of fluorophore-doped glass- or polymerbased slides and/or slides with fluorescent coatings for the spectral region of $c a .450$ to $900 \mathrm{~nm}$ that show ideally little photodecomposition under application-relevant conditions and a homogeneous distribution of a single or a mixture of different fluorophores. The albeit stated photo-stability should be given in terms of applicationrelevant light fluxes at typically used excitation wavelengths. A more single-use type approach could include for instance the application of slide-type cells or capillaries with a defined optical pathlength filled with (renewable) fluorophore solutions. With respect to the desired standardization of microscopic measurements, the application-relevant spectroscopic properties of these potential reference materials, that need to be tested with a broad variety of commercially available fluorescence microscopes, should be preferably certified [37].

\section{Fluorescence- and Biology-Related Problems in the Microarray Technology}

Biochips have become an increasingly important research tool for the biotechnology industry, molecular diagnostics, and related fields of gene expression analysis, drug screening, nucleic acid sequencing, and mutation analysis [114-119]. Such biochips consist of a plurality of binding agents or so-called probes. Such probes can be peptides, oligonucleotides, or up to ten thousands of single stranded DNA fragments representing a single gene or genome that are deposited onto the surface of a solid support like a coated glass slide in the form of an array with spot sizes in the 50 to $200 \mu \mathrm{m}$ range. For typical DNA microarray experiments with fluorescence detection, RNA or DNA from biological samples and from controls is isolated and - during a reverse transcription reaction-that transcribes RNA into cDNA-labeled with two spectrally distinguishable dyes like the cyanine dyes Cy3 (absorption at $c a .550 \mathrm{~nm}$, emission at $c a .565 \mathrm{~nm}$ ) and Cy5 (absorption at $c a .650 \mathrm{~nm}$, emission at $c a .675 \mathrm{~nm}$ ), re- spectively. The labeled biomolecules are then reacted, i.e. hybridized with the microarray thereby binding to their complementary sequences present in the spotted probes. After processing, the microarrays are read out with an array reader, typically a confocal laser scanner equipped with a $\mathrm{He}-\mathrm{Ne}$ laser $(543,5,594,612$, and $632.8 \mathrm{~nm})$ or a $\mathrm{Kr}-\mathrm{Ar}$ laser $(488,514,568$, and $647 \mathrm{~nm})$ or less common, a solid-state laser or a white light source and a PMT or a CCD (charge coupled device) detection system with a lateral resolution of 5-10 $\mu \mathrm{m}$, respectively. This step involves excitation at two different wavelengths and subsequent recording of integral fluorescence intensities in two detection channels, the so-called green and red channel [120]. Usually, the scanner software is able to combine signal intensities of both channels and to deliver a false-color image. The amount of analyte bound to a spot follows from the measured integral fluorescence intensity with abundant targets seen either in red or green and equal amounts in yellow. This allows for the estimation of relative expression levels of the genes from the fluorescence intensities of the spots. For the generation of reliable gene expression data, several repetitions and controls are necessary with the obtained expression values being statistically analyzed and normalized. Further bioinformatic analysis, e.g. hierarchical agglomerative clustering of the gene expression data, provides the basis for the interpretation of the alteration in gene expression of many genes in parallel [121-123].

Microarray experiments are performed either by ratio or direct measurements comparing two RNA samples, e.g. healthy and disease stage, or by absolute or indirect measurements comparing one RNA sample to a reference, e.g. standardized RNA or DNA. Accordingly, this yields either relative expression values (ratios) or absolute expression values. To improve the reliability of the data, suitable controls are introduced that are incorporated into every experiment and are accordingly present on the array and spiked into the hybridization solution. This includes range, ratio, negative, and contamination controls as well as a positive control and a guide-dot for orientation after scanning. As an example for both approaches, image files of the two different experimental designs measuring direct (A) and indirect (B) gene expression values of the plant Arabidopsis thaliana during its growth are shown in Fig. 11, respectively. For the former, RNA from two different stages are labeled either with $\mathrm{Cy} 3$ or Cy5 and hybridized to an Arabidopsis microarray. For indirect measurement of gene expression values, the target sample is labeled either with $\mathrm{Cy} 5$ or $\mathrm{Cy} 3$, and a vice versa labeled reference oligonucleotide that is complementary to the PCR primers used for probe generation is spiked into the hybridization solution and co-hybridized. 

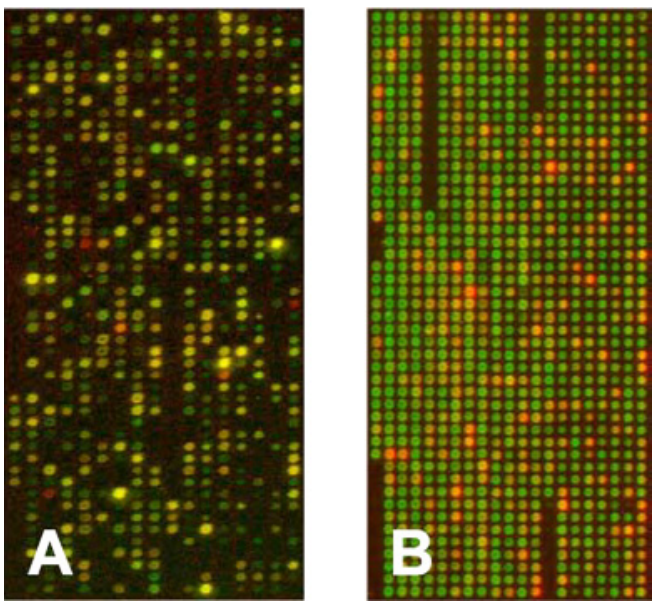

Fig. 11. Image files of two different experimental designs that enable the comparison of gene expression between samples from Arabidopsis thaliana growth development. For direct (ratio) measurement of differences in gene expression, see (A), RNA from two different developmental stages were labeled either with $\mathrm{Cy} 3$ or $\mathrm{Cy} 5$ and hybridized to an Arabidopsis microarray, respectively. For indirect (absolute) measurement, see (B), RNA from one developmental stage was labeled with Cy5 and the sample was co-hybridized with a Cy3-labeled reference oligonucleotide and measured as described before. The microarrays were scanned at excitation wavelengths of 532 and $635 \mathrm{~nm}$, respectively, and read out with the respective green and red channel with a resolution of $10 \mu \mathrm{m}$ using a Fuji FLA 8000 microarray scanner. Only sections of the corresponding images are shown.

The uncertainty and reliability of the results obtained from microarray experiments depend on the biological and the experimental variation as well as - to a smaller extent —on fluorescence detection and data analysis. Furthermore, due to the lack of generally accepted standardized sampling protocols and biological standards to be used in every microarray experiment, problems related to biology and data analysis are amplified [124]. In general, the contribution to the overall uncertainty from the biological side has the highest impact due to genetic and environmental variation, which are difficult to measure. Fluorescence-inherent sources of error can be easier certified, however only, if the necessary controls and standards are included in the experiment and if the scanners are properly characterized. Fluorescence-based uncertainties are related to fluorophore labeling of the target $[125,126]$ and to problems linked to measurements of fluorescence intensities, i.e. the sensitivity of the label's spectroscopic properties to chromophore microenvironment $[127,128]$ and to dye-dye interactions [129] as well as to instrumentspecific effects. To overcome limitations in the former, e.g. different incorporation rates of the dyes or dye instability, the so-called dye swap or fluor-reverse is used with both targets being labeled with two dyes in sepa- rate reactions and hybridized to two identical microarrays with identical probe arrangements. In addition to the instrument-related effects already discussed, microarray scanner-specific uncertainties are linked to the determination of the background signal, the method used for dye normalization, crosstalk between channels, and variations in the alignment of the optical scanner [130]. For example, for confocal reading, which is used to increase the sensitivity by rejecting light coming from other planes than that of the biological signals of interest, there is a strong need for accuracy of the focus and tilt adjustment of the biochip. To illustrate the influence of such instrumentspecific effects on the recorded fluorescence intensities, a calibration slide from Clondiag [131] was measured with three common microarray readers with routinely used instrument parameters, e.g. representative settings of the PMT voltage for the green and red channels, respectively. The accordingly obtained results are depicted in Fig. 12. The differences in fluorescence intensity measured with all the readers for the two detection channels are attributed

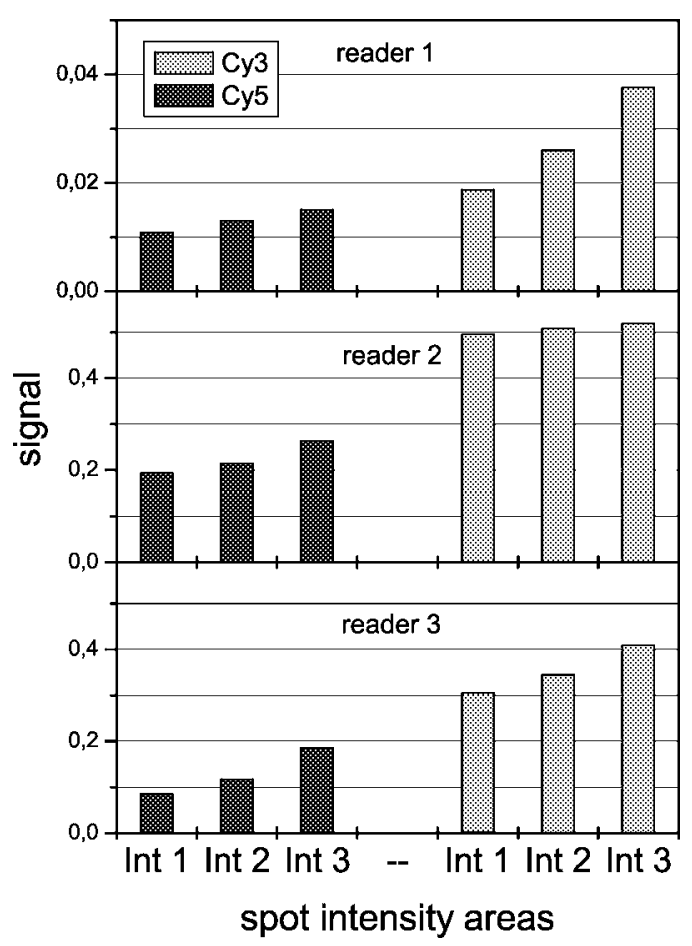

Fig. 12. Comparison of three commercially available microarray readers using the calibration slide FluorIS from Clondiag, Germany. The intensities of three areas differing in intensity (Int 1, Int 2, and Int 3) are depicted. The measured intensities represent mean values of 100 single spots per spot area. The slide was measured at the excitation wavelengths typically used for Cy3 (light-gray bars) and Cy5 (dark-grey bars) at standard laboratory PMT settings. The obtained scan images were analyzed using the IconoClust version 2.2 software package (Clondiag, Germany). 
to the different wavelength-dependent spectral responsivities of the respective detection systems, see e.g. also Fig. 1. Typically, such detection systems are less sensitive in the long wavelength region of $c a .675 \mathrm{~nm}$ as compared to a detection wavelength of $c a .565 \mathrm{~nm}$. The obvious deviations between the three data sets clearly demonstrate the need for a general calibration tool to improve the reliability of microarray data from the detection side.

To make microarray data world-wide comparable, there is a strong need for instrument standards, application of biological reference materials, and standardized procedures/protocols for the performance and evaluation of such experiments. For the reduction of biology- and data evaluation-related uncertainties, the microarray research community has discussed since a long time the introduction of quality controls (QCs) and SOPs as well as biological reference materials such as for instance standards based on genomic DNA, a reference RNA pool or a reference oligonucleotide for measuring more precisely absolute or indirect gene expression values [132,133]. For instance, only recently, two RNA reference materials have been defined to improve the standardization of gene expression experiments [134]. Furthermore, working groups have been founded to develop and share common standards and guidelines for gene expression analysis in clinical trials and to cover standards for reference materials and quality control, SOPs as well as standard bioinformatic approaches for data analysis and storage [135-138]. Several guidelines for the performance of microarray experiments have been proposed by the Microarray Gene Expression Data (MGED) Society, including the Minimal Information About a Microarray Experiment (MIAME) description and annotation [139] and the MAGE-ML mark-up language [140]. For storage of the microarray data, several databases have been established following the MIAME guidelines [141,142].

To improve the reliability of microarray experiments from the detection side, at present, there exist only very few standards. These are intensity standards designed e.g. for control of the performance of array scanners, channel crosstalk, and effect of PMT voltage as well as for the comparison of the performance of different instruments [143]. One of them offers some means for control of the spatial resolution [131]. This standard, however, that was measured by us, see Fig. 12, suffers from photodegradation, lack of reported absorption and emission spectra, and a comparatively small variation in fluorescence intensity, see for instance [131] and Fig. 12, which does not allow for the determination of the linearity of the detection system(s). Moreover, some microarray reader manufacturer have integrated a self-test using an internal light source, which runs at the start of the instruments and before every scan (e.g. Fuji Photo Film Co.) to check PMT settings whereas others (e.g. Axon Instruments Inc.) provide specific calibration slides for an evaluation of the instrument performance.

To improve this situation, better-suited calibration slides or calibration spots on every microarray are desired to help adjust the scanner settings and to determine the instrument's image/spatial resolution as well as its day-to-day performance and long-term stabilityideally in combination with guidelines for their use. Furthermore, tested procedures in combination with recommended materials/systems are needed for the determination of the range of linearity of the array scanners, their dynamic range and sensitivity. The final goal is here to improve the comparability of data generated by different instruments/laboratories and with different labels. Easyto-use fluorescence standards that meet these requirements should for instance either provide a stable output at application-relevant wavelengths and photon fluxes or need to be easily reproduced, should have minimum local and global non-uniformities in emission and should generate signals comparable to those observed for typical samples. With this respect, also the influence of spectral deviations between the fluorescence spectra of the standard and common labels needs to be examined. This renders the availability of absorption and corrected emission spectra of both the standard and the labels for applicationrelevant conditions, i.e. microenvironment(s), important. For testing of the instrument's spatial resolution, which should be in the $5 \mu \mathrm{m}$ range, uniformly patterned standards are desired. Such standards must be applicable for the majority of typically used microarray readers and labels, e.g. suited for typical excitation wavelengths and emission wavelength intervals as well as scanner formats. Moreover, they should have been tested with a broad variety of instruments in a Round Robin test. For the development of such purpose-fit fluorescence standards, input not only from instrument manufacturers but also from the community of users of the microarray technology is required. Additionally, future technological advances of microarray readers should be considered that will most likely provide an enhanced spatial resolution, precision, and sensitivity as well as a higher dynamic range. Furthermore, it needs to be decided on within the community of users of the microarray technology and instrument manufacturers, whether stand-alone fluorescence standards like calibration slides or the integration of fluorescence standards into slides used for the performance of microarray experiments are to be favored. The former is for instance sufficient to characterize the day-to-day and long-term instrument performance, whereas the latter approachthough more costly—may be tempting for an improved 
and better feasible comparability of the recorded fluorescence signals.

\section{In Vivo Fluorescence Imaging/Spectroscopy}

Generally, imaging of animals, human skin, and inner cavities within the human body, e.g. bladder or colon, with endoscopic techniques is a powerful tool to detect modifications of tissue, possibly in advance of malignant alterations. Apart from reflection measurements, application of fluorescence imaging in medical diagnostics has attracted increasing interest over the past decade [144,145]. Since tissue is a highly scattering medium, the excitation light as well as the light emitted by the fluorophore used as contrast agent are diffracted many times and accordingly, the corresponding optical path-lengths are significantly increased. Contributions from light emitted from different depths within the tissue result in a diffuse image and hence, the technique is often termed diffuse fluorescence imaging. Optical properties of tissue strongly depend on excitation and emission wavelengths. Accordingly, wavelength selection influences the penetration depth and, hence, the intensity of the recorded fluorescence signal. In particular, the maximum penetration depth and minimum absorption are reached when excitation and emission wavelengths are chosen that fall within the so-called diagnostic and therapeutic window between 700 and $900 \mathrm{~nm}$. Hence, this spectral region is used for the detection of deeper lying lesions. For the detection of superficial lesions, the wavelength region between 400 and $700 \mathrm{~nm}$ is employed as well.

In vivo fluorescence imaging allows for instance to study the dynamics of contrast agents or specific intrinsic fluorophores, which serve as reporters for diseased tissue [146]. In addition, fluorescence measurements based on specific molecular probes for target detection, i.e. molecular imaging, are used in medicine and drug design [147152]. Fluorescence detection typically includes measurements of the distribution of fluorescence intensities (integral, spectrally resolved, time-gated) of the corresponding reporter molecules as well as fluorescence lifetime and occasionally fluorescence polarization analysis. The former is strongly influenced by the absorption of the respective tissue, its light scattering properties, and the location/depth of the fluorophores in the tissue. In addition, apart from the fluorescence of the probe molecule, background fluorescence of tissue caused by intrinsic chromophores, i.e. autofluorescence, also contributes to the fluorescence signal. Furthermore, for fluorescence originating from deeper layers within the tissue, the covering tissue acts as wavelength-dependent attenuator for the excitation light as well as for the emitted light. Also reflec- tion losses, which might depend on the polarization of light, occur at boundary layers (e.g. mucosa) because of the inhomogeneity of tissue. Accordingly, many influencing quantities have to be known to derive concentrations of fluorophores and subsequently the concentration of target molecules from the observed fluorescence signals. At present, this intention seems to be unrealistic. However, standardization of measurement procedures to obtain relative concentrations at higher precision is required to ensure the comparability of measurements performed with different setups and/or under different experimental conditions in vivo. The application of suitable SOPs and reference materials would facilitate the interpretation of fluorescence images. In particular, the differentiation between a lesion and normal tissue might be improved in this way.

Imaging of laser-induced fluorescence in tissue can be performed in reflection [153] or transmission geometry [154]. In both cases, a reference material with known optical, i.e. absorption, fluorescence, and scattering properties can be measured simultaneously or subsequently using the same geometry. A suited non-certified reference material developed by the PTB for this purpose consists of a cyanine dye and glass spheres as scattering centers embedded into a polymer matrix [155]. We used this reference material to derive the results shown in Fig. 13, which depicts the measured integral fluorescence intensities from a rat joint and fluorescence intensities normalized to this standard detected simultaneously. The contrast agent, a nonspecific cyanine dye $[151,152,156]$ injected intravenously prior to the experiment, was excited at $740 \mathrm{~nm}$. Apart from the large penetration depth, for the chosen excitation wavelength, the background fluorescence is very

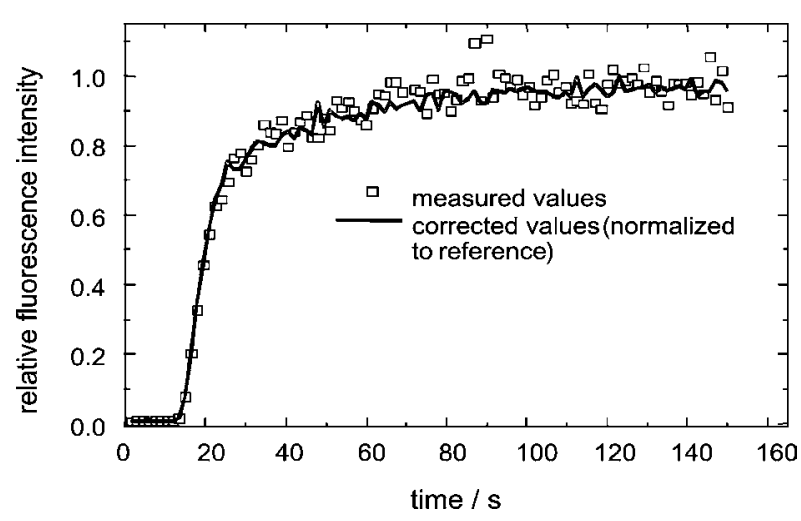

Fig. 13. Comparison of measured (squares) and normalized (line) fluorescence intensities of a rat joint after application of the contrast agent (non-specific cyanine dye, dose of $1 \mu \mathrm{mol} / \mathrm{kg}$ body weight). The normalization was done with respect to a PTB-developed reference material simultaneously recorded. The contrast agent was excited at $740 \mathrm{~nm}$. 
low compared to the fluorescence intensity of the contrast agent, see Fig. 13, observation times shorter than $15 \mathrm{~s}$. The background fluorescence from the tissue is obtained by imaging the animal before application of the contrast agent. As follows from Fig. 13, the integral fluorescence intensity, plotted versus time after intravenous injection, strongly increases between 15 and $25 \mathrm{~s}$ and reaches a saturation value after about $90 \mathrm{~s}$. Furthermore, the fluctuations in the bolus kinetic are considerably reduced on application of this normalization procedure as is evident from the reduced signal variation. The remaining variation of the fluorescence signal is associated with the heartbeat and respiration of the animal, respectively. This referencing or normalization procedure allows to compare fluorescence intensities from different animals provided the normalization is performed with the same reference material.

Fluorescence imaging and fluorescence spectroscopy can be used to extend the potential of endoscopybased techniques frequently used for clinical applications. As an example, in Fig. 14, an endoscopic image of the colon mucosa of a patient with pancolitis is shown. Aside from the typical reflection image routinely observed, a bright spot from the excitation light coupled through an optical fiber and the fiber tip can be seen. To obtain laser-induced fluorescence, aminolevulinic acid (ALA) is sprayed into the colon shortly before endoscopy. ALA is a poorly fluorescing precursor of the fluorophore proto-

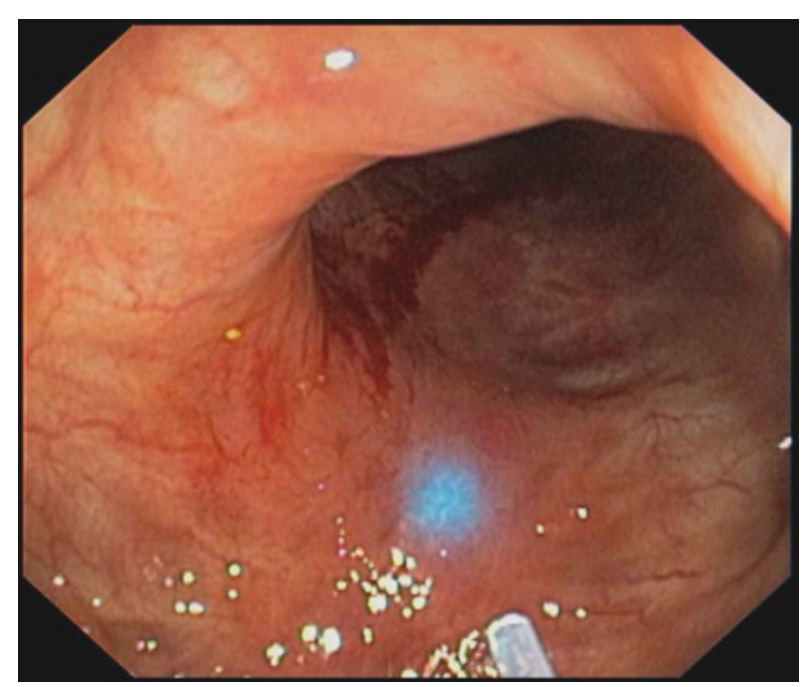

Fig. 14. Fluorescence-guided endoscopy of the colon of a patient with pancolitis. The laser light delivered by a fiber and the fiber tip can be recognized in the lower central part of the image. This investigation has been performed at the 4th Medical Department, Charité, Humboldt University, Berlin, Germany by Dr. G. Schachschal.

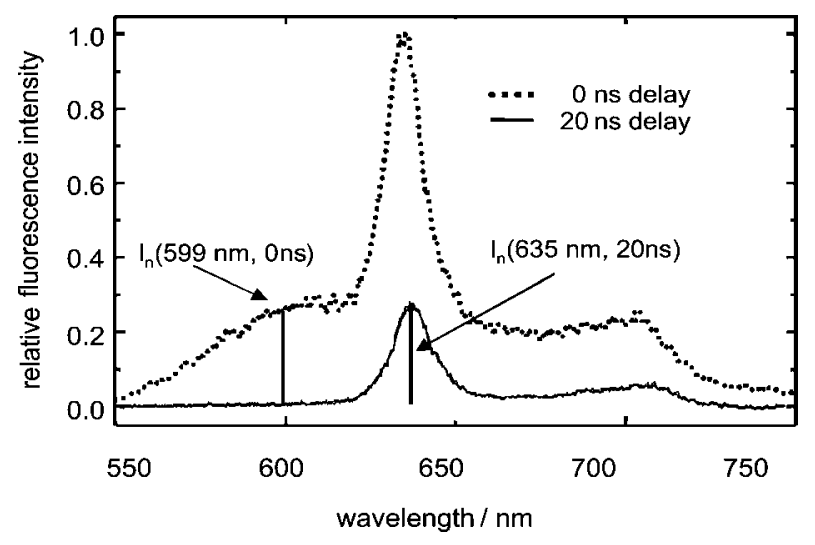

Fig. 15. Prompt and delayed fluorescence spectrum of a dysplasia in Barrett's oesophagus. The background fluorescence is efficiently suppressed in the time delayed spectrum of PpIX. The arrows indicate the wavelengths and amplitudes used for normalization.

porphyrin IX (PpIX), the formation of which is enhanced in diseased tissue, e.g. in low- and high-grade dysplasia. To monitor regions with enhanced PpIX concentration on-line together with the conventional endoscopic image, pulsed laser light for the excitation of the fluorophore and a monochromator coupled to an intensified photodiode array for the detection of the emitted light are used. For excitation and observation of laser-induced fluorescence an optical fiber is inserted into the working channel of an endoscope. This technique enables the measurement of spectrally resolved fluorescence data at a single point $[157,158]$ and can be applied for fluorescence-guided biopsy. The fluorescence spectra from the blue spot in Fig. 14 taken at two different delay times relative to the exciting laser pulse are illustrated in Fig. 15. As is evident from a comparison of the prompt and delayed emission spectra, application of this time-gated technique results in an effective suppression of tissue autofluorescence. Since geometrical factors remain unchanged, analysis of both spectra can be exploited to eliminate geometrical effects, which otherwise hamper the interpretation and quantification of the measurement. To this end [158], we introduce the $\mathrm{P}_{\mathrm{p}} \mathrm{IX}$-specific quantity

$$
\begin{aligned}
I_{\mathrm{n}}^{\mathrm{sp}}(\lambda, 20 \mathrm{~ns})= & I_{\mathrm{n}}(599 \mathrm{~nm}, 20 \mathrm{~ns})\left\{\frac{I_{\mathrm{n}}(\lambda, 20 \mathrm{~ns})}{I_{\mathrm{n}}(599 \mathrm{~nm}, 20 \mathrm{~ns})}\right. \\
& \left.-\frac{I_{\mathrm{n}}(\lambda, 0 \mathrm{~ns})}{I_{\mathrm{n}}(599 \mathrm{~nm}, 0 \mathrm{~ns})}\right\}
\end{aligned}
$$

In Eq. (1), the prompt $I(\lambda, 0 \mathrm{~ns})$ and delayed $I(\lambda$, $20 \mathrm{~ns})$ fluorescence spectra, see Fig. 15 are normalized to the maximum intensity $I(635 \mathrm{~nm}, 0 \mathrm{~ns})$ of the prompt fluorescence spectrum. The resulting normalized quantities are indicated by the lower index $n$. The normalization to 
$I(635 \mathrm{~nm}, 0 \mathrm{~ns})$ is chosen with respect to the maximum emission of PpIX and the division by the spectral amplitude at $\lambda=599 \mathrm{~nm}$ is performed because non-specific fluorescence and PpIX fluorescence spectra do not overlap in this region. In this way, the contribution from nonspecific autofluorescence can be subtracted, provided that the optical properties of diseased and normal tissue are similar. This assumption is generally justified for the detection of early stages of cancerous tissue. On the basis of this normalization procedure, a significant discrimination between diseased and non-diseased tissue is possible as has been shown for example by Ortner et al. for dysplasias in Barrett's oesophagus [157].

For fluorescence imaging in vivo, for spectral correction as well as for the determination of the linearity of the detection system, its dynamic range, and its sensitivity, solutions of the corresponding contrast agents or other spectrally suited dyes could be used. However, to improve the comparability and reliability of in vivo applications of fluorescence imaging, on our opinion, the use of reference procedures including mathematical analysis and properly chosen fluorescence standards seems to be the method of choice, as has been demonstrated exemplary for molecular imaging experiments with small animals and fluorescence endoscopy of human colon. Reference materials suited for signal quantification must also mimic the scattering properties of the investigated tissue. To this end, for the comparability of different in vivo applications, a set of well-characterized and preferably certified standards of liquid or solid nature is desired that covers the spectral range from 400 up to $900 \mathrm{~nm}$.

\section{CONCLUSION AND OUTLOOK}

As has been illustrated for selected fluorescence techniques, there is still a considerable need to improve the reliability and the comparability of fluorescence data as well as the comparability of instrument characterization and performance validation. The latter forms the basis for standardization. To achieve this and to improve quality assurance in fluorometry, well-characterized instrument-type and application-specific fluorescence standards for the UV/Vis/NIR spectral region are mandatory, that closely match the optical properties of measured samples. In this context, close resemblance stands for comparable absorption and emission features, emission intensities and characteristics, scattering and measurement geometry. In addition to (certified) reference materials, internationally accepted SOPs for the characterization and validation of common types of fluorescence instruments are needed as well as guidelines for the performance of fluorescence measurements that are relevant for a broad community of users of fluorescence techniques. The intention of the latter should be also to reduce sample-related and handlingrelated uncertainties in fluorescence analysis. On our opinion, guidelines-preferably in combination with suited materials or standards - are for instance required for the determination of the range of linearity, dynamic range, and sensitivity of fluorescence instruments as well as for the performance of quantitative fluorescence measurements and for the determination of fluorescence quantum yields. The availability of suited certified standards and guidelines is of special importance for fluorescence measurements in medical diagnosis and clinical applications of fluorometry, where standardization of instrument characterization and performance of measurements is eventually requested.

Novel fluorescence standards for the establishment of an improved quality assurance in fluorometry on a broad level should be not only designed and characterized for use under application-relevant conditions, easy-to-operate, and commercially available, but also characterized with respect to all the application-relevant spectroscopic and analytical properties. Furthermore, the (wavelength-dependent) uncertainty of the calibrationrelevant fluorometric quantity/quantities needs to be provided including method of determination. To guarantee the standard's reliability, such reference materials should be preferably certified. The rational design and testing of such fluorescence standards with improved properties and the development of the aforementioned guidelines, however, requires a close contact between National Metrological Institutes (NMIs), standardization bodies, regulatory agencies, scientific associations, manufacturers of instrumentation, users of fluorescence techniques as well as manufacturers of fluorescent labels, reagents and other materials for fluorescence analysis. Within this context, also the necessary level of traceability in fluorometry has to be defined and recent developments in instrumentation as well as in fluorescent dyes need to be taken into account. Particular attention should be focused also on procedures for the evaluation of software used for data analysis, at least for more advanced fluorescence methods like time-resolved fluorometry, fluorescence imaging, and fluorescence-based microarray techniques.

\section{ACKNOWLEDGMENTS}

Financial support from the German Ministry of Economics and Labor (BMWA) is gratefully acknowledged (BMWA VI A2-17/03). We thank Mrs. A. Hoffmann and Mrs. M. Spieles for technical assistance, Dr. D. Pfeifer for 
stimulating discussions and Dr. J. Enderlein for carefully reading the section on fluorescence microscopy.

\section{REFERENCES}

1. J. R. Lakowicz (Ed.) (1999). Principles of Fluorescence Spectroscopy, 2nd edn., Kluwer Academic/Plenum Press, New York.

2. J. R. Lakowicz (Ed.) (1992-2004). Topics in Fluorescence Spectroscopy Series, Vols. 1-8, Plenum Press, New York.

3. O. S. Wolfbeis (Series Ed.) (2001-2004). Springer Series on Fluorescence, Methods and Applications, Vols. 1-3, Springer, Berlin.

4. S. G. Schulman (Ed.) (1985-1993). Molecular Luminescence Spectroscopy Parts, Vols. 1-3, Wiley Interscience, New York.

5. W. T. Mason (1999). Fluorescent and Luminescent Probes for Biological Activity, 2nd edn., Academic Press, San Diego.

6. A. J. Pope, U. M. Haupts, and K. J. Moore (1999). Homogenous fluorescence readouts for miniaturized high-throughput screening: Theory and practice. Drug Discov. Today 4(8), 350-362.

7. E. Zubritsky (1999). Microplate readers reach critical mass. Anal. Chem. News Features 71, 39A-43A.

8. C. T. Wittwer, K. M. Ririe, R. V. Andrew, D. A. David, R. A. Gundry, and U. J. Balis (1997). The light cycler: A microvolume multisample fluorimeter with rapid temperature control. Biotechniques 22, 176-181.

9. Supplement (1998). Fluoreszenzspektroskopie. Nachr. Chem. Tech. Lab. 46, S121-S132.

10. Analytical Methods Committee (1998). Evaluation of analytical instrumentation. Part XI Instrumentation for molecular fluorescence spectrometry. Analyst 123, 1649-1656.

11. J. W. Eastman (1966). Standardization of fluorescence spectra and the calibration of spectrofluorimeters. Appl. Optics 5(7), 11251132 .

12. W. Galbraith, K. W. Ryan, N. Gliksman, D. L. Taylor, and A. S. Waggoner (1989). Multiple spectral parameter imaging in quantitative fluorescence microscopy. I: Quantitation of bead standards. Comput. Med. Imag. Graph. 13, 47-60.

13. A. K. Gaigalas, L. Li, O. Henderson, R. Vogt, J. Barr, G. Marti, J. Weaver, and A. Schwartz (2001). The development of fluorescence intensity standards. J. Res. Natl. Inst. Stand. Technol. 106(2), 381389.

14. C. A. Parker (1968). Photoluminescence of Solutions, Elsevier, Amsterdam.

15. J. N. Miller (1981). Standards in Fluorescence Spectrometry, U1traviolett Spectrometry Group, London.

16. D. F. Eaton (1988). Reference materials for fluorescence measurement. Pure Appl. Chem. 60, 1107-1114.

17. R. A. Velapoldi and M. S. Epstein (1989). In M. C. Goldberg (Ed.), Luminescence Applications in Biological, Chemical, Environmental and Hydrological Sciences, ACS Symposium Series, Vol. 383, American Chemical Society, Washington, DC, pp. 98126.

18. W. D. Niles and F. S. Cohen (1995). Radiometric calibration of a video fluorescence microscope for the quantitative imaging of resonance energy transfer. Rev. Sci. Instrum. 66, 35273536.

19. EN ISO/IEC 17025; GLP/GMP, GLP: Good laboratory praxis; GMP: Good manufacturing praxis.

20. Burgess and D. G. Jones (1995). Spectrophotometry, Luminescence and Colour: Science and Compliance, Elsevier, Amsterdam.

21. O. D. D. Soares and J. L. C. Costa (1999). Spectrophotometers intercomparison for spectrocolorimetric scale harmonization. Rev. Sci. Instrum. 70(12), 4471-4481.

22. J. C. Travis, J. C. Zwinkels, F. Mercader, A. Ruiz, E. A. Early, M. V. Smith, M. Noel, M. Maley, G. W. Kramer, K. L. Eckerle, and D. L. Duewer (2002). An international evaluation of holmium oxide solution reference materials for wavelength calibration in molecular absorption spectrophotometry. Anal. Chem. 74, 34083415.

23. ASTM E 169-87 (reapproved 2003). Standard Practices for general Techniques of Ultraviolet-Visible Quantitative Analysis, and herein referenced ASTM standards.

24. CIE-Publ. 15.2, Colorimetry, 2nd edn., 1986.

25. K. D. Mielenz (1987). In C. Burgess and K. D. Mielenz (Eds.), Advances in Standards and Methodology in Spectrophotometry, Elsevier, Amsterdam, pp. 49-62.

26. D. C. Rich and D. Martin (1999). Improved model for improving the inter-instrument agreement of spectrocolorimeters. Anal. Chim. Acta 380, 263-276.

27. K. Witt (2002/2003). Haben wir die UV/vis-Spektrometerie lumineszierender Materialien im Griff? Die Farbe 46(1/2), 31-51.

28. ASTM E 388-72 (reapproved 2003). Spectral bandwidth and wavelength accuracy of fluorescence spectrometers.

29. ASTM E 578-83 (reapproved 2003). Linearity of fluorescence measuring system.

30. ASTM E 579-84 (reapproved 2003). Limit of detection of fluorescence of quinine sulfate.

31. A. Schwartz and E. Fernandez-Repollet (1993). Development of clinical standards for flow cytometry. Ann. N. Y. Acad. Sci. 677, 28-39.

32. A. Schwartz, M. Mendez, G. Santiago, L. Diaz, and E. FernandezRepollet (1997). Applications of common quantitative fluorescent standards to multiple platforms: Comparison of commercial fluorescent calibration standards used in quantitative flow cytometry. Clin. Immunol. 17(1), 14-18.

33. A. Schwartz, G. E. Marti, J. W. Gratama, and E. Fernandez-Repollet (1998). Standardizing flow cytometry: A classification system of fluorescence standards used for flow cytometry. Cytometry 33, 106114.

34. J. W. Gratama, J. L. D'Hautcourt, F. Mandy, G. Rothe, D. Barnett, G. Janossy, S. Papa, G. Schmitz, and R. Lenkei (1998). Flow cytometric quantitation of immunofluorescence intensity: Problems and perspectives. Cytometry 33, 166-178.

35. R. A. Velapoldi (1987). Liquid standards in fluorescence spectrometry. In C. Burgess and K. D. Mielenz (Eds.), Advances in Standards and Methodology in Spectrophotometry, Elsevier, Amsterdam, pp. 175-193.

36. I. Billard, E. Ansoborlo, K. Afferson, S. Arpigny, M. E. Azenha, D. Brich, P. Bros, H. D. Burrows, G. Choppin, L. Couston, V. Dubois, T. Fanghänel, G. Geipel, S. Hubert, J. I. Kim, T. Kimura, R. Klenze, A. Kronenberg, M. Kumke, G. Lagarde, G. Llamarque, S. Lis, C. Madic, G. Meinrath, C. Moulin, R. Nagaishi, D. Parker, G. Plancque, F. Scherbaum, E. Simoni, S. Sinkov, and C. Viallesoubranne (2003). Appl. Spectrsc. 57(8), 1027-1038.

37. For clear definition of the metrological hierarchy of reference materials, see International Vocabulary of Basics and General Terms in Metrology (1994), 2nd edn., Beuth Verlag GmbH.

38. R. A. Velapoldi and K. D. Mielenz (1980). A fluorescence standard reference material: Quinine sulfate dihydrate, NBS Spec.Publ. 260264, PB 80132046, Springfield, VA.

39. A. Schwartz, L. Wang, E. Early, A. Gaigalas, Y.-Z. Zhang, G. E. Marti, and R. F. Vogt (2002). Quantitating fluorescence intensity from fluorophore: The definition of MESF assignment. J. Res. Natl. Inst. Stand. Technol. 107(1), 83-91.

40. U. Resch-Genger, D. Pfeifer, C. Monte, W. Pilz, A. Hoffmann, M. Spieles, J. Hollandt, R. D. Taubert, B. Schönenberger, P. Nording (in press), Traceability of fluorometry. Part II: Spectral fluorescence standards. J. Fluoresc. 15(3), 325.

41. J. W. Verhoeven (1996). Molecular terms used in photochemistry (recommendations 1996). Pure Appl. Chem. 68(12), 2223-2286.

42. J. Hollandt, R. D. Taubert, J. Seidel, A. Gugg-Helminger, U. ReschGenger, D. Pfeifer, C. Monte, and W. Pilz (in press), Traceability of fluorometry. Part I: Physical standards. J. Fluoresc. 15(3), 311.

43. E. D. Cehelnik, K. D. Mielenz, and R. A. Velapoldi (1975). Polarization effects on fluorescence measurements. J. Res. Natl. Bur. Stand. A 79A(1), 1-15. 
44. U. Resch-Genger, K. Hoffmann, and A. Engel, manuscript in preparation.

45. T. Erdogan, A. Pradhan, and V. Mizrahi (2003). Optical filters impact fluorescence fidelity. Biophotonics Int. 10(10), 38-43.

46. J.W. Hofstraat and M. J. Latuhihin (1994). Correction of fluorescence spectra. Appl. Spectrosc. 48(4), 436-446.

47. J. A. Gardecki and M. Maroncelli (1998). Set of secondary emission standards for calibration of the spectral responsivity in emission spectroscopy. Appl. Spectrosc. 52(9), 1179-1189.

48. R. J. Kovach and W. M. Peterson (1994). The measurement of sensitivity in fluorescence spectroscopy. Am. Lab. 32G-32K.

49. R. B. Thompson, I. Gryczynski, and J. Malicka (2002). Fluorescence polarization standards for high-throughput screening and imaging. Biotechniques 32(1), 34-41.

50. See for instance Molecular Probes, Starna GmbH, Matech Precision Dynamics Coorp., Labsphere Inc., Fluka GmbH, LambdaChem GmbH, and SUMITA Optical Glass Inc. as well as NIST (RM 8640, SRM 936a, and 1932).

51. R. F. Chen (1972). Measurement of absolute values in biochemical fluorescence spectroscopy. J. Res. Nat. Bur. Stand. A 76(6), 593606.

52. R. B. Thompson, I. Gryczynski, and J. Malicka (2002). Fluorescence polarization standards for high-throughput screening and imaging. Biotechniques 32(1), 34-41.

53. I. T. Lifshotz and M. L. Meilman (1989). Standard sample for calibrating wavelength scales of spectral fluorimeters. Sov. J. Opt. Technol. 55(8), 487-489.

54. S. A. Wise, L. C. Sander, and W. E. May (1993). Determination of polycyclic aromatic hydrocarbons by liquid chromatography. J. Chromatogr. 642, 329-349.

55. SRM $1647 \mathrm{~b}$, NIST.

56. A. Schwartz, A. K. Gaigalas, L. Wang, G. E. Marti, R. F. Vogt, and E. Fernandez-Repollet (2004). Formalization of the MESF unit of fluorescence intensity. Cytometry 57B(1), 1-6.

57. RM 8640, NIST.

58. P. Froehlich (1989). Under the sensitivity specification for a fluorescence spectrophotometer. Int. Lab. 42-44.

59. R. J. Kovach and W. M. Peterson (1994). The measurement of mensitivity in fluorescence spectroscopy. Am. Lab. 32G-32K.

60. ISO (1993). Guide to the expression of uncertainty in measurement.

61. J. N. Demas (1982). In K. D. Mielenz (Ed.), Optical Radiation Measurements, Vol. 3, Academic Press, New York, p. 195.

62. W. Geffken (1962). The molar absorption of different ions in glasses. Glastechn. Berichte 35, 27-35.

63. M. Mizuguchi, H. Hosono, and H. Kawazone (1999). Timeresolved photoluminescence for diagnostic of photoluminescence to ArF excimer laser damage to $\mathrm{CaF}_{2}$ single crystals. J. Opt. Soc. Am. 7(16), 1153-1159.

64. P. de Rose (2003). Bioanalytical and biomedical applications of fluorescence techniques: Instrument characterization and validation, traceability, and need for reference materials, in Fluorescence Workshop, BERM-9, Berlin.

65. D. Ehrt, P. Ebeling, U. Natura, U. Kohlberg, K. Naumann, and S. Ritter (2001). Redox equilibria and ultraviolet radiation induced defects in glasses. Int. Cong. Glass 1, 84-96.

66. J. W. Chan, T. Huster, J. S. Hayden, S. H. Risbud, and D. M. Krol (2002). J. Am. Ceram. Soc. 85(5), 1037-1040.

67. A. Engel, K. Knapp, B. Speit, G. Wehrhan, and E. Mörsen (2001). High quality $\mathrm{CaF}_{2}$ used for $157 \mathrm{~nm}$ micro lithography. Fab. Tech. 14, 177-184.

68. C. Mühlig, W. Triebel, G. Töpfer, and A. Jordanov (2003). CaF 2 for ArF lithography-Characterisation by in-situ and LIF measurements, CHOCLAB II final report-Optics Characterization, pp. 257-267.

69. A. Engel, W. Triebel, C. Mühlig, J. Alkemper, A. Krämer, J. Kandler, K. Knapp, and E. Mörsen (2000). Visualization of laser damage in $157 \mathrm{~nm}$ material $\mathrm{CaF}_{2}$ and $\mathrm{BaF}_{2}, 1$ st $157 \mathrm{~nm}$ Symposium, Dana Point CA, pp. 391-398.
70. A. Engel, R. Haspel, and V. Rupertus (2003). Advanced industrial fluorescence metrology used for qualification of high-quality optical materials. SPIE Proc. 5118-5120, 182-189.

71. R. A. Velapoldi (1971). Fluorescence. Nat. Bur. Stand. Tech. Note 584, 53-83.

72. H. Pick (1972). Structure of trapped electron and trapped hole centers in alkali halides. In J. Abeles (Ed.), Optical Properties of Solids, North Holland, pp. 653-668.

73. D. W. Pack, W. J. Manthey, and D. S. Mc Clure (1989). Production of color centers with ionizing irradiation in alkali halides. Phys. Rev. B 40(14), 9930-9935.

74. M. Letz, A. Engel, L. Parthier, U. Natura, and K. Knapp (2004). $\mathrm{CaF}_{2}$ for DUV lens fabrication: Basic material properties and dynamic light-matter interaction. SPIE Proc. 5377, Optical Microlithography XVII, 1797-1804.

75. Japanese Optical Glass Industrial Standards, JOGIS, 03-1975.

76. W. Gellermann (1989). J. Chem. Solids 52, 249-254.

77. W. Goehde, U. Cassens, L. G. Lehman, Y. Traore, W. Goehde jun., P. Berkes, C. Westerberg, and B. Greve (2003). Individual patient-dependent influence of erythrocyte lysing procedures on flow-cytometric analysis of leukocyte subpopulations. Transfusion Med. Hemother. 30, 165-170.

78. J.-C. Strohmeyer, C. Blume, C. Meisel, W.-D. Doecke, M. Hummel, C. Hoeflich, K. Thiele, A. Unbehaun, R. Hetzer, and H.-D. Volk (2003). Standardized immune monitoring for the prediction on infections after cardiopulmonary bypass surgery in risk patients. Cytometry 53B, 54-62.

79. G. Monneret, N. Elmenkouri, J. Bohe, A. L. Debard, A. C. Gutowski, J. Bienvenu, and A. Lepape (2002). Analytical requirements for measuring monocytic human lymphocyte antigen DR by flow cytometry: Application to the monitoring of patients with septic shock. Clin. Chem. 48, 1589-1592.

80. S. B. Iyer, M. J. E. Bishop, B. Abrams, V. C. Maino, A. J. Ward, T. P. Christion, and K. A. Davis, QuantiBRITE ${ }^{\text {TM }}$ : A new standard for fluorescence quantification, www.bdbiosciences.com/ immunocytometry_systems (see download literature, White Papers, QuantiBRITE $^{\mathrm{TM}}$ White Paper).

81. Y. Gerena-López, J. Nolan, L. Wang, A. Gaigalas, A. Schwartz, and E. Fernández-Repollet (2004). Quantification of EGFP expression on Molt-4 T cells using calibration standards. Cytometry $\mathbf{6 0 A}, 21-$ 28.

82. J. B. Pawley (Ed.) (1995). Handbook of Biological Confocal Microscopy, 2nd edn., Kluwer Academic Publishers, New York.

83. S. Inoue (Ed.) (1986). Video Microscopy, Plenum Publishers, New York.

84. D. B. Murphy (Ed.) (2001). Fundamentals of Light Microscopy and Electronic Imaging, Wiley-Liss, New York.

85. X. F. Wang, A. Periasamy, B. Herman, and D. M. Coleman (1992). Fluorescence lifetime imaging microscopy (FLIM): Instrumentation and applications. Crit. Rev. Anal. Chem. 23(5), 369395.

86. Special issue on the use of ion-sensitive fluorophores for making accurate intracellular ion measurements at high spatial and/or temporal resolution (1990). Cell Calcium, February/March.

87. M. Andreeff and D. Pinkel (Ed.) (1999). Introduction to Fluorescence in Situ Hybridization: Principals and Clinical Applications, Wiley-Liss, New York.

88. V. E. Centonze, A. Takahashi, E. Casanova, and B. Herman (2000). Quantitative fluorescence microscopy. J. Histotechnol. 23(3), 229234.

89. W. D. Niles and F. C. Cohen (1995). Radiometric calibration of a video fluorescence microscope for quantitative imaging of resonance energy transfer. Rev. Sci. Instrum. 66(6), 3527-3536.

90. R. Nitschke (2004). Standardization and quantification in microscopy, Workshop AK PhotonicNet, Wetzlar.

91. The point-spread function is determined by the product of the excitation intensity distribution and the light collection efficiency function. 
92. J. S. Ploem (1970). Standards for fluorescence microscopy, in E. J. Holborow (Ed.), Standard for Immunofluorescence Symposium, Blackwell Scientific Publications, Oxford, pp. 137-153.

93. W. Galbraith, K. W. Ryan, N. Gliksman, D. Lansing Taylor, and A. S. Waggoner (1989). Multiple spectral parameter imaging in quantitative fluorescence microscopy. I: Quantitation of bead standards. Comp. Med. Imag. Graphics 13(1), 47-60.

94. J. M. Lerner and R. M. Zucker (2004). Calibration and validation of confocal spectral imaging systems. Cytometry 62A, 8-34.

95. M. Sernetz and A. Thaer (1970). A capillary fluorescence standard for microfluorometry. J. Microscopy 91(1), 43-52.

96. F. W. D. Rost (1991). Quantitative Fluorescence Microscopy, Cambridge University Press, Cambridge, p. 236.

97. D. S. Kaplan and G. L. Picciolo (1989). Characterization of instrumentation and calibrators for quantitative microfluorometry for immunofluorescence tests. J. Clin. Microbiol. 27, 442-447.

98. A. P. M. Jongsma, W. Hijmans, and J. S. Ploem (1971). Quantitative immunofluorescence. Histochemie 25, 329-343.

99. R. A. Velapoldi, J. C. Travis, W. A. Cassatt, and W. T. Yap (1975). Inorganic ion-doped glass fibres as microspectrofluorimetric standards. J. Microsc. 103(3), 293-303.

100. R. P. Haugland (Ed.) (2002). Handbook of Fluorescent Probes and Research Products, 9th edn., Molecular Probes, Section 24.1.

101. J. J. Haaijman and J. P. R. van Dalen (1974). Quantification in immunofluorescence microscopy: A new standard for fluorescein and rhodamine emission measurement. J. Immunol. Methods $\mathbf{5}$, 359-374.

102. S. J. Lockett, K. Jacobson, and B. Herman (1992). Quantitative precision of an automated fluorescence-based image cytometer. Anal. Quant. Cytol. Histol. 14, 187-202.

103. M. A. Model and J. K. Burkhardt (2001). A standard for calibration and shading correction of a fluorescence microscope. Cytometry $\mathbf{4 4 ,}$ 309-316.

104. J. E. Sisken (1989). Fluorescent standards. Methods Cell Biol. 30, 113-126.

105. S. G. Turney, S. M. Culican, and J. W. Lichtman (1996). A quantitative fluorescence-imaging technique for studying acetylcholine receptor turnover at neuromuscular junctions in living animals. J. Neurosci. Methods 64, 199-208.

106. P. W. Stevens and D. M. Kelso (2003). Imaging and analysis of immobilized particle arrays. Anal. Chem. 75, 1147-1154.

107. A. C. Jones, M. Millington, J. Muhl, J. M. De Freitas, J. S. Barton, and G. Gregory (2001). Calibration of an optical fluorescence method for film thickness measurement. Meas. Sci. Technol. 12, N23-N27.

108. Evident Technologies, Product Catalogue, February 2004.

109. A. P. Alivisatos (1996). Semiconductor clusters, nanocrystals, and quantum dots. Science 271, 934-937.

110. A. Knight, J. Gaunt, T. Davidson, V. Chechik, and S. Windsor (2004). Evaluation of the suitability of quantum dots as fluorescence standards. NPL report DQL-AS 007.

111. W. G. J. H. M. van Sark, P. L. T. M. Frederix, D. J. van den Heuvel, H. C. Gerritsen, A. A. Bol, J. N. J. van Lingen, C. de Mello Donega, and A. Meijerink (2001). Photoxidation and photobleaching of single CdSe/ZnS quantum dots probed by room-temperature time resolved spectroscopy. J. Phys. Chem. B 105(10), 82818284.

112. I. T. Young (1983). The use of digital image processing techniques for the calibration of quantitative microscopes. Proc. SPIE 387, 326-335.

113. APE GmbH, Berlin; www.ape-berlin.de.

114. M. C. Pirrung (2002). How to make a DNA chip. Angew. Chem. Int. Ed. 41(8), 1276-1289.

115. J. R. Epstein, I. Biran, and D. R. Walt (2002). Fluorescence-based nucleic acid detection and microarrays. Anal. Chim. Acta 469, 336.

116. B. Lemieux, A. Asharoni, and M. Schena (1998). Overview of DNA chip technology. Mol. Breed. 4, 277-289.
117. P. Hedge, R. Qi, K. Abernathy, C. Gay, S. Dharap, R. Gaspard, J. E. Hughes, E. Snesrud, N. Lee, and J. Quackenbush (2000). A concise guide to cDNA microarray analysis. Biotechniques 29(3), $548-562$.

118. C. B. V. Christensen (2002). Arrays in biological and chemical analysis. Talanta 56, 289-299.

119. Biochips (Market survey) (2003). New Drugs 26-29.

120. M. Schena, D. Shalon, R. W. Davis, and P. O. Brown (1995). Quantitative monitoring of gene expression patterns with a complementary DNA microarray. Science 270(5235), 467-470.

121. M. B. Eisen, P. T. Spellman, P. O. Brown, and D. Botstein (1998). Cluster analysis and display of genome-wide expression patterns. Proc. Natl. Acad. Sci. U.S.A. 95(25), 14863-14868.

122. S. Granjeaud, F. Bertucci, and B. R. Jordan (1999). Expression profiling: DNA arrays in many guises. Bioassays 21(9), 781-790.

123. M. B. Eisen and P. O. Brown (1999). DNA arrays for analysis of gene expression. Meth. Enzymol. 303, 179-205.

124. A. Butte (2002). The use and analysis of microarray data. Nat. Rev. Drug Discov. 1, 951-960

125. M. Taniguchi, K. Miura, H. Iwao, and S. Yamanaka (2001). Quantitative assessment of DNA microarrays-Comparison with Northern Blot analyses. Genomics 71, 34-39.

126. M. Bartosiewicz, M. Trounstine, D. Barker, R. Jonston, and A. Buckpitt (2000). Development of a toxicological gene array and quantitative assessment of this technology. Arch.Biochem. Biophys. 376(1), 66-73.

127. E. A. Winzeler, M. Schena, and R. W. Davis (1999). Fluorescencebased expression monitoring using microarrays. Meth. Enzymol. 306, 3-18.

128. Z. Guo, R. A. Guilfoyle, A. J. Thiel, R. Wang, and L. M. Smith (1994). Direct fluorescence analysis of genetic polymorphism by hybridization with oligonucleotide arrays an glass supports. $\mathrm{Nu}$ cleic Acids Res. 22(24), 5456-5465.

129. J. B. Randolph and A. S. Waggoner (1997). Stability, specifity and fluorescence brightness of multiply-labeled fluorescent DNA probes. Nucleic Acids Res. 25(14), 2923-2929.

130. F. Perraut, A. Lagrange, P. Pouteau, O. Peyssonneaux, P. Puget, G. McGall, L. Menou, R. Gonzalez, P. Labeye, and F. Ginot (2002). A new generation of scanners for DNA chips. Biosens. Bioelectron. 17, 803-813.

131. K. Adelheim, E. Emantraut, T. Kaiser, and J. Tuchscheerer (2002). Smart chip for array experiment standardization. New Drugs 2223.

132. A. M. Dudley, J. Aach, M. A. Steffen, and G. M. Church (2002). Measuring absolute expression with microarrays with a calibrated reference sample and an extended signal intensity range. Proc. Natl. Acad. Sci. U.S.A. 99(11), 7554-7559.

133. M. R. Weil, T. Macatee, and H. R. Garner (2002). Toward a universal standard: Comparing two methods for standardizing spotted microarray data. Biotechniques 32(6), 1310-1314.

134. M. Cronin, K. Ghosh, F. Sistare, J. Quackenbush, V. Vilker, and C. O'Connell (2004). Universal RNA Reference Materials for Gene Expression. Clin. Chem. [meeting review].

135. Y. F. Leung and D. Cavalieri (2003). Fundamentals of cDNA microarray data analysis. Trends Genet. 19(11), 649-659.

136. T. Forster, D. Roy, and P. Ghazal (2003). Experiments using microarray technology: Limitations and standard operating procedures. J. Endocrinol. 178(2), 195-204.

137. Tumor Analysis Best Practices Working Group (2004). Expression profiling - Best practices for data generation and interpretation in clinical trials. Nat. Rev. Genet. 5(3), 229-237.

138. A. T. Weeraratna, J. E. Nagel, V. d. V. Mello-Coelho, and D. D. Taub (2004). Gene expression profiling: from microarrays to medicine. J. Clin. Immunol. 24(3), 213-224.

139. A. Brazma, P. Hingamp, J. Quackenbush, G. Sherlock, P. Spellman, C. Stoeckert, J. Aach, W. Ansorge, C. A. Ball, H. C. Causton, T. Gaasterland, P. Glenisson, F. C. Holstege, I. F. Kim, V. Markowitz, J. C. Matese, H. Parkinson, A. Robinson, U. Sarkans, S. Schulze- 
Kremer, J. Stewart, R. Taylor, and J. Vilo (2001). Vingron M. Minimum information about a microarray experiment (MIAME)toward standards for microarray data. Nat. Genet. 29(4), 365-371.

140. P. T. Spellman, M. Miller, J. Stewart, C. Troup, U. Sarkans, S. Chervitz, D. Bernhart, G. Sherlock, C. Ball, M. Lepage, M. Swiatek, W. L. Marks, J. Goncalves, S. Markel, D. Iordan, M. Shojatalab, A. Pizarro, J. White, R. Hubley, E. Deutsch, M. Senger, B. J. Aronow, A. Robinson, D. Bassett, C. J. Jr. Stoeckert, and A. Brazma (2002). Design and implementation of microarray gene expression markup language (MAGE-ML). Genome Biol. 3(9), RESEARCH0046.

141. A. Brazma, H. Parkinson, U. Sarkans, M. Shojatalab, J. Vilo, N. Abeygunawardena, E. Holloway, M. Kapushesky, P. Kemmeren, G. G. Lara, A. Oezcimen, P. Rocca-Serra, and S. A. Sansone (2003). ArrayExpress-A public repository for microarray gene expression data at the EBI. Nucleic Acids Res. 31(1), 68-71.

142. J. Gollub, C. A. Ball, G. Binkley, J. Demeter, D. B. Finkelstein, J. M. Hebert, T. Hernandez-Boussard, H. Jin, M. Kaloper, J. C. Matese, M. Schroeder, P. O. Brown, D. Botstein, and G. Sherlock (2003). The Stanford Microarray Database: Data access and quality assessment tools. Nucleic Acids Res. 31(1), 94-96.

143. See for example, Clondiag Chip Technologies GmbH; Full Moon Biosystems Inc.

144. G. A. Wagnières, W. M. Star, and B. C. Wilson (1998). In vivo fluorescence spectroscopy and imaging for oncological applications. Photochem. Photobiol. 68, 603-632.

145. R. Richards-Kortum and E. M. Sevick-Muraca (1996). Quantitative optical spectroscopy for tissue diagnosis. Annu. Rev. Phys. Chem. 47, 555-606.

146. D. J. Cruccia, F. Bevilacqua, A. J. Durkin, S. Merritt, B. J. Tromberg, G. Gulsen, H. Yu, J. Wang, and O. Nalcioglu (2003). In vivo quantification of optical contrast agent dynamics in rat tumors by use of diffuse optical spectroscopy with magnetic resonance imaging coregistration. Appl. Opt. 42, 2940-2950.

147. D. Y. Paithankar, A. U. Chen, B. W. Pogue, M. S. Patterson, and E. M. Sevick-Muraca (1997). Imaging of fluorescent yield and lifetime from multiply scattered light reemitted from random media. Appl. Opt. 36, 2260-2272.
148. R. Weissleder and U. Mahmood (2001). Mol. Imag. Radiol. 219, 316-333.

149. V. Ntziachristos, J. Ripoll, and R. Weissleder (2002). Would nearinfrared fluorescence signals propagate through large human organs for clinical studies? Opt. Lett. 27, 333-335.

150. R. Weissleder and V. Ntziachristos (2003). Shedding light onto live molecular targets. Nat. Med. 9, 123-128.

151. M. Rudin and R. Weissleder (2003). Molecular imaging in drug discovery and development. Nat. Rev. Drug Discov. 2, 123131.

152. V. Ntziachhristos, C. Bremer, and R. Weissleder (2003). Fluorescence imaging with near-infrared light: New technological advances that enable in vivo molecular imaging. Eur. Radiol. 13, 195-208.

153. A. Becker, C. Hessenius, K. Licha, B. Ebert, U. Sukowski, W. Semmler, B. Wiedenmann, and C. Grötzinger (2001). Receptortargeted optical imaging of tumors with near-infrared fluorescent ligands. Nat. Biotechnol. 19, 327-331.

154. E. E. Graves, J. P. Culver, J. Ripoll, R. Weissleder, and V. Ntziachristos (2004). Singular-value analysis and optimization of experimental parameters in fluorescence molecular tomography. J. Opt. Soc. Am. A Opt. Image Sci. Vis. 21, 231-241.

155. U. Sukowski, F. Schubert, D. Grosenick, and H. Rinneberg (1996) Preparation of solid phantoms with defined scattering and absorption properties for optical tomography. Phys. Med. Biol. 41, 18231844.

156. K. Licha (2002). Contrast agents for optical imaging. Topics Curr. Chem. 222, 22-29.

157. M.-A. E. J. Ortner, B. Ebert, E. Hein, K. Zumbusch, D. Nolte, U. Sukowski, J. Weber-Eibel, B. Fleige, M. Dietel, M. Stolte, G. Oberhuber, R. Porschen, B. Klump, H. Hörtnagel, H. Lochs, and H. Rinneberg (2003). Time gated fluorescence spectroscopy in Barrett's oesophagus. Gut 52, 28-33.

158. K. T. Moesta, B. Ebert, T. Handke, D. Nolte, C. Nowak, W. E. Haensch, R. K. Pandey, T. J. Dougherty, H. Rinneberg, and P. M. Schlag (2001). Protoporphyrin IX occurs naturally in colorectal cancers and their metastases. Cancer Res. 61, 991999. 